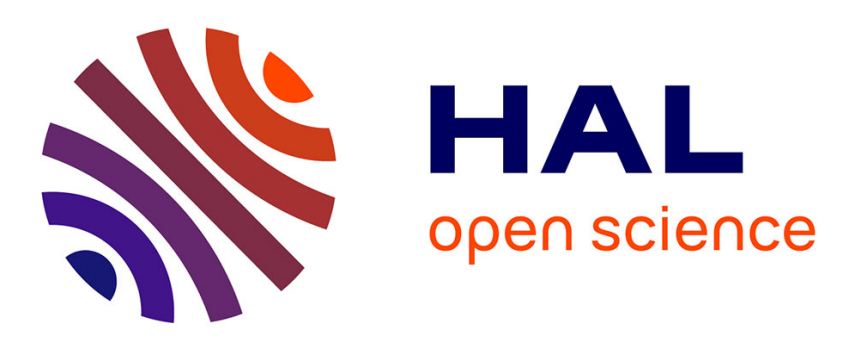

\title{
A Sliding Mode Controller for a Model of Flow Separation in Boundary Layers
}

\author{
Tonametl Sanchez, Andrey Polyakov, Jean-Pierre Richard
}

\section{To cite this version:}

Tonametl Sanchez, Andrey Polyakov, Jean-Pierre Richard. A Sliding Mode Controller for a Model of Flow Separation in Boundary Layers. International Journal of Robust and Nonlinear Control, 2020, 30 (3), pp.1181-1202. 10.1002/rnc.4822 . hal-02373078

\section{HAL Id: hal-02373078 \\ https://hal.inria.fr/hal-02373078}

Submitted on 20 Nov 2019

HAL is a multi-disciplinary open access archive for the deposit and dissemination of scientific research documents, whether they are published or not. The documents may come from teaching and research institutions in France or abroad, or from public or private research centers.
L'archive ouverte pluridisciplinaire $\mathbf{H A L}$, est destinée au dépôt et à la diffusion de documents scientifiques de niveau recherche, publiés ou non, émanant des établissements d'enseignement et de recherche français ou étrangers, des laboratoires publics ou privés. 


\title{
A Sliding Mode Controller for a Model of Flow Separation in Boundary Layers
}

\author{
Tonametl Sanchez ${ }^{1}$, Andrey Polyakov ${ }^{1}$, and Jean-Pierre Richard ${ }^{2}$ \\ ${ }^{1}$ Inria, Univ. Lille, CNRS, UMR 9189 - CRIStAL \\ ${ }^{2}$ Inria, Univ. Lille, École Centrale de Lille, CNRS, UMR 9189 - CRIStAL
}

\begin{abstract}
In this paper we propose a sliding mode controller for a MIMO model of flow separation in boundary layers. The model consists in a bilinear system with constant delays in both the state and the input. The main motivation to consider such a class of systems is that, it has shown to be suitable for input-output modelling and control design of some turbulent flow control systems. Stability and robustness properties of the control scheme are studied by means of Volterra equations theory, which provides easily verifiable stability conditions.
\end{abstract}

\section{Introduction}

Control of turbulent flows in boundary layers is a fundamental problem in several technological applications, e.g. transportation systems as road vehicles or aircraft. For example, an important concern in a vehicle design is the drag, which is significantly increased by the flow separation phenomenon [13, 2, 3] (see Fig. 1). Thus, improvements in control of turbulent flows can produce important benefits, e.g. reduction of drag and fuel consumption in vehicles. [13, 2] However, existing techniques of active flow control find several barriers to be applied in practice. On one hand, the classical model for flow is given by the Navier-Stokes equations which appear to be too much complex for control design purposes. For instance, the implementation of an observer-based controller that requires to solve the Navier-Stokes model online is not realistic because of the high computational cost. [2] On the other hand, model approximations by finite-dimensional linear systems can be more appropriate for control design or implementation. Unfortunately, many relevant features of the flow are not well reproduced by such linear models, which restricts the corresponding controllers to a very narrow range of situations. A thorough survey about the active control techniques for flow systems can be found in the work of Brunton and Noack[2].

A simplified input-output nonlinear model for flow separation control systems was proposed in the work of Feingesicht et al. [7] That model succeeded in reproducing the 

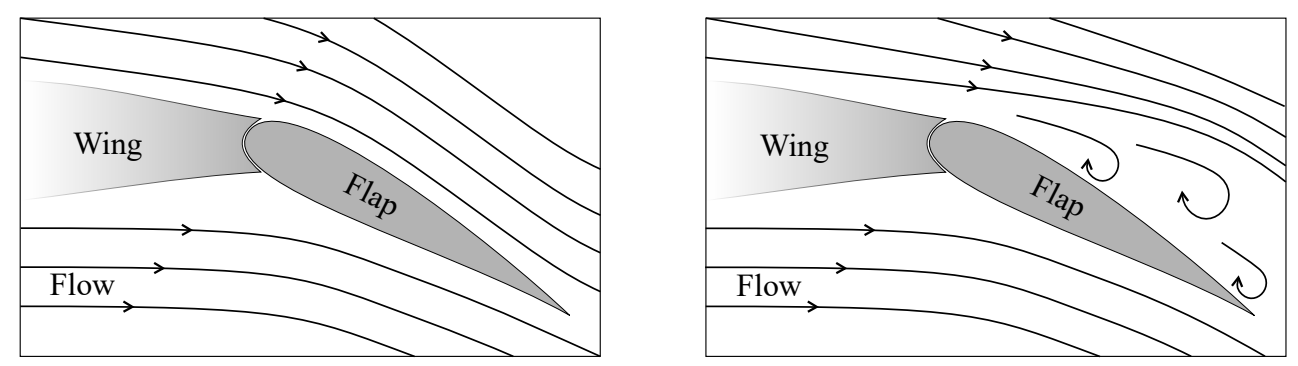

Figure 1: Schematic diagram of a wing-flap configuration moving through a flow. Left: ideal flow. Right: separated flow (with recirculation bubbles).

input-output behaviour of the system in different experimental configurations with a low margin error, and with only a few parameters to identify. [5] Such a model consists in a bilinear differential equation with delays in the input and in the state. The argument to propose that kind of equations as models for flow systems was presented in Feingesicht. [5] There, it was shown that, under certain assumptions, a spatial discretisation of a controlled Burgers' equation (which is a unidimensional approximation of the Navier-Stokes equations) produces a bilinear time-delay differential equation.

For a particular case of the model introduced by Feingesicht et al., 7] a sliding mode controller was proposed in Feingesicht et al. [6] That control technique was chosen due to the on-off nature of the synthetic jet actuators. A good experimental performance was obtained with such a controller in different experimental configurations. [6, 3] Unfortunately, the controllers proposed by Feingesicht et al. [6] and Sanchez et al. [19] are limited to SISO systems, and their generalization to the MIMO case is not straightforward.

In this paper we consider a class of MIMO bilinear systems (with constant delays in both the state and the input) as an input-output model for flow separation control systems, generalizing that one proposed in the work of Feingesicht et al. [7] In terms of the flow control application, considering multiple outputs constitutes an exciting issue, since it allows for dealing with 3-dimensional flow behaviours, such as non-symmetrical recirculation bubbles behind vehicles, to be controlled thanks to several air-jet blowers with various places and orientations. In terms of the control theory, extending the design of a sliding mode control scheme from SISO to MIMO systems is also challenging: 1) the model includes linear and bilinear interconnection terms that must be taken into account in the control design; 2) the nature of the actuators demands bounded control signals; 3) for the sliding mode control, the way to propose the sliding variable is not straightforward from the SISO case since the interconnection terms have to be considered, and even when a sliding variable has been designed, it has to be proven that the sliding regime is established despite the interaction with the other subsystems; 4) some restrictions on the model (derived from the physical system), as boundedness and positivity of the solutions, have to be taken into account for the design of the controller.

We want to emphasize that the existing control techniques for delayed or bilinear systems do not adapt well to the class of systems considered in this paper, for example: 
the standard techniques to control bilinear systems neglect the delays in the state and the input, and usually do not consider restricted controllers[14, 15]; bilinear systems with constrained control are studied in [11, but the delayed case is not considered; in [21], sliding modes and delays in the state are considered, but the system is linear in the input which is not delayed and not restricted; bilinear (discrete-time) systems are considered in [22] but the control is not restricted and the delay in the state is not included in the analysis. The only similar approach is the switched controller given in [18], however, there are some important differences between that contribution and our proposed controller: 1) the classes of systems, although similar, are different regarding the conditions imposed on the admissible state and input delays, e.g. in [18 all the inputs must have the same delay, in contrast, different delays in the inputs are allowed in the present paper; 2) asymptotic stability is easier to verify with the method proposed in this paper, no linear matrix inequality has to be solved as in [18]; 3) the robustness analysis made in this paper is simple, however in [18], no robustness analysis is made; 4) the proposed sliding mode controller can be regarded as a decentralized control, while the switched approach is a centralized controller.

The sliding mode technique proposed in this paper takes into account all the restrictions derived from the physical plant (on-off actuators, boundedness and positivity of solutions, and input delays) to guarantee the convergence of the trajectories of the model to the reference points. Thus, a main theoretical contribution of this paper is the proposed sliding mode control design methodology for bilinear systems with state and input delays. Let us now list some features of the sliding mode controller proposed in this paper:

- the controller guarantees the convergence of the trajectories of the model to the reference points, which means a reduction of the flow separation in the flow system;

- the conditions to guarantee the stability and robustness properties of the control scheme are easily verifiable;

- the stability and robustness properties of the sliding dynamics (which is infinite dimensional and described by an integral equation) are analysed by means of Volterra integral equations theory (as it was done for the SISO case by Sanchez et al. [19]);

- the structure of the controller demands a low computational cost;

- the methodology to design the controller can be applied on other physical plants modelled by MIMO bilinear systems with delays;

- the controller can be applied to control the SISO systems considered in Feingesicht et al. [6] and Sanchez et al. [19].

Paper organization: In Section 2 we describe the system's model and the control problem. Some stability properties of the model are studied in Section 3. The sliding mode controller is shown in Section 4, and its stability proof is given in Section 5. The robustness of the proposed scheme is analysed in Section 6. A simulation example is shown in Section 7. Concluding remarks and future work are stated in Section 8. 


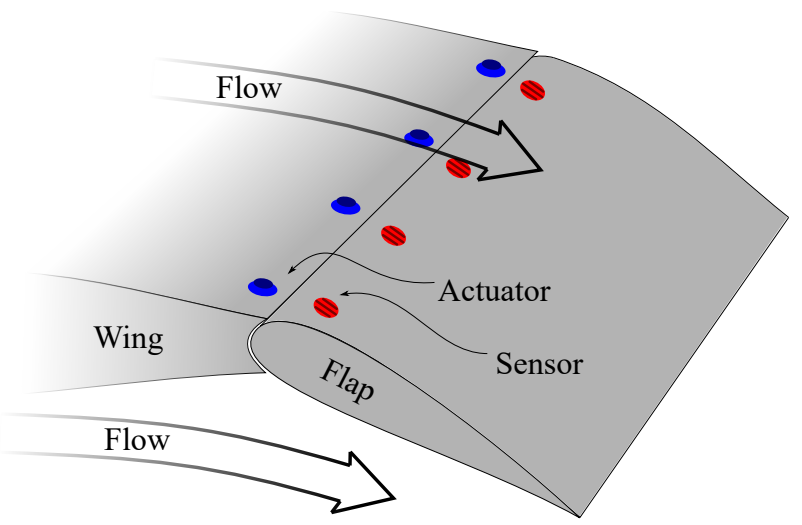

Figure 2: A flow separation control scheme in a wing-flap configuration.

Notation: The sets of real and integer numbers are denoted by $\mathbb{R}$ and $\mathbb{Z}$, respectively. For any $a \in \mathbb{R}, \mathbb{R}_{\geq a}$ denotes the set $\{x \in \mathbb{R}: x \geq a\}$, and analogously for $\mathbb{R}_{>a}$. For any $p \in \mathbb{R}_{\geq 1}, L^{p}(\bar{J})$ denotes the set of Lebesgue-measurable functions $x: J \subset \mathbb{R} \rightarrow \mathbb{R}$ with finite norm $\|x\|_{L^{p}(J)}=\left(\int_{J}|x(s)|^{p} \mathrm{~d} s\right)^{1 / p}$, and $L^{\infty}(J)$ denotes the set of Lebesgue-

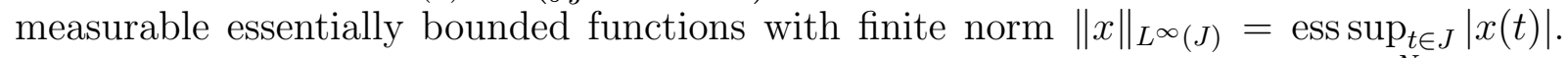
For sums (when the limits are clear from the context) we use: $\sum_{k}$ to denote $\sum_{k=1}^{N} ; \sum_{k \neq i}$ to denote $\sum_{\substack{k=1 \\ k \neq i}}^{N}$; and $\sum_{k, s}$ to denote $\sum_{k=1}^{N} \sum_{s=1}^{M}$, e.g. $\sum_{k \neq i, s}$ denotes $\sum_{\substack{k=1 \\ k \neq i}}^{N} \sum_{s=1}^{M}$.

\section{Description of the model and problem statement}

Fig. 1 shows a schematic diagram of the section of a wing-flap configuration moving through a flow. The flow separation phenomenon is represented on the right of Fig. 1: it is an important source of loss of lift and increment of drag. To perform active control, the system can be equipped with actuators that alter the flow, and with sensors that measure such alteration, as depicted in Fig. 2. In several experimental configurations, the actuators and sensors consist in pneumatic valves (also known as synthetic jets or air blowers) and surface thermal anemometers (also known as hot-film sensors), respectively. [2, 5] If we consider only one pair of actuator-sensor in Fig. 2, then we obtain a SISO system from the input $u$ to the output $y$, see the schematic diagram shown in Fig. 3 (left). For such a SISO system, the following model was proposed in the work of Feingesicht et al., 6]

$$
\dot{x}(t)=\sum_{k=1}^{N_{1}} a_{k} x\left(t-\tau_{k}\right)+\left(\sum_{s=1}^{N_{2}} \bar{a}_{s} x\left(t-\bar{\tau}_{s}\right)+b\right) u(t-\varsigma),
$$

for some finite $N_{1}, N_{2} \in \mathbb{Z}_{>0}$. This model has been validated in different experimental configurations. 5] However, the simple example in Fig. 2 shows several pairs of actuatorsensor, thus it constitutes a MIMO system. In this case, (1) could be used repeatedly on each pair of actuator-sensor along the wing to model the whole system. Nevertheless, the main disadvantage of such a procedure is that it does not consider the interaction 


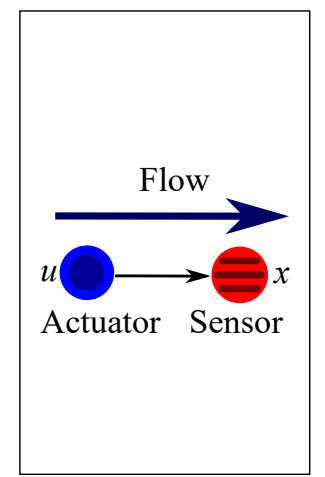

SISO

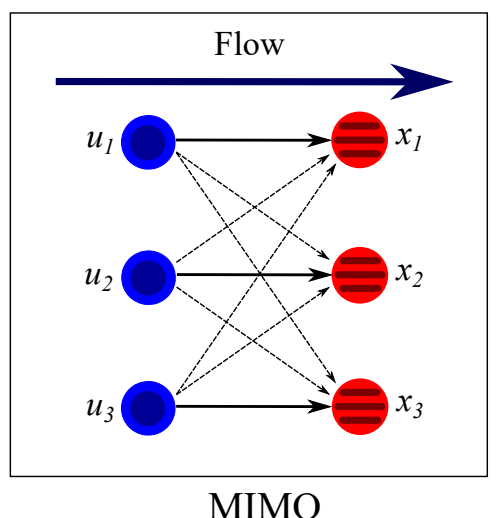

MIMO

Figure 3: Simplified schematic diagrams of the control system for the SISO and MIMO cases.

between subsystems. A more realistic situation is depicted in the grid of actuator-sensor pairs shown in Fig. 3 (right). In this paper, inspired by such applications which need to generalize (1), we propose an input-output model for such a MIMO configuration. Let $n \in \mathbb{Z}_{>0}$ be the number of pairs of actuator-sensor. For $i \in\{1, \ldots, n\}$, denote with $u_{i}$ and $x_{i}$ the $i$-th input and output, respectively. Thus, we consider the following system of bilinear time-delay differential equation: $\mathrm{s}^{1}$

$$
\begin{aligned}
\dot{x}_{i}(t)= & \sum_{k} a_{i}^{k} x_{i}\left(t-\tau_{i}^{k}\right)+\left(\sum_{s} \bar{a}_{i}^{s} x_{i}\left(t-\bar{\tau}_{i}^{s}\right)+b_{i}\right) u_{i}\left(t-\varsigma_{i}\right)+ \\
& \sum_{s, r \neq i} \bar{a}_{i}^{s, r} u_{r}\left(t-\varsigma_{i}^{s, r}\right) x_{i}\left(t-\bar{\tau}_{i}^{s}\right)+R_{i}(t),
\end{aligned}
$$

where, for some finite $N_{i}^{1}, N_{i}^{2} \in \mathbb{Z}_{>0}, k \in\left\{1, \ldots, N_{i}^{1}\right\}, s \in\left\{1, \ldots, N_{i}^{2}\right\}$, and $r \in\{1, \ldots, n\}$. The coefficients $a_{i}^{k}, \bar{a}_{i}^{s}, b_{i}, \bar{a}_{i}^{s, r} \in \mathbb{R}$, and the delays $\tau_{i}^{k}, \bar{\tau}_{i}^{s}, \varsigma_{i}, \varsigma_{i}^{s, r} \in \mathbb{R}_{\geq 0}$. The terms in the second line of (2) represent the interconnection between subsystems. Note that if in (2) $R_{i}=0$ and $\bar{a}_{i}^{s, r}=0$ for all $s, r$, then we recover (1). In this paper, we consider two cases for the term $R_{i}$.

Case 1. For each $i \in\{1, \ldots, n\}$, the term $R_{i}$ is a known time signal such that $R_{i} \in$ $L^{\infty}\left(\mathbb{R}_{\geq 0}\right)$. In this case, the only coupling terms (that depend on the state) are those whose coefficients are $\bar{a}_{i}^{s, r}$.

Case 2. For each $i \in\{1, \ldots, n\}$, the term $R_{i}$ has the following bilinear structure

$$
R_{i}(t):=\sum_{j \neq i, k} a_{i j}^{k} x_{j}\left(t-\tau_{i j}^{k}\right)+\sum_{j \neq i, s^{\prime}, r} u_{r}\left(t-\varsigma_{i j}^{s^{\prime}, r}\right) \bar{a}_{i j}^{s^{\prime}, r} x_{j}\left(t-\bar{\tau}_{i j}^{s^{\prime}}\right)+\sum_{j \neq i} b_{i j} u_{j}\left(t-\varsigma_{i j}\right),
$$

${ }^{1}$ Note that, due to the multiple indexes in the model, we are using both subscripts and superscripts as indexes in the coefficients and delays. 
where $j \in\{1, \ldots, n\}$, and for some finite $N_{i}^{3} \in \mathbb{Z}_{>0}, s^{\prime} \in\left\{1, \ldots, N_{i}^{3}\right\}$. The coefficients $a_{i j}^{k}, \bar{a}_{i j}^{s^{\prime}, r}, b_{i j} \in \mathbb{R}_{\geq 0}$, and the delays $\tau_{i j}^{k}, \varsigma_{i j}^{s^{\prime}, r}, \bar{\tau}_{i j}^{s^{\prime}}, \varsigma_{i j} \in \mathbb{R}_{\geq 0}$.

Remark 1. In this paper we assume that the parameters of the model are known.2 and we focus on the control design. Although the parameters and the interconnection terms in (2) are assumed to be perfectly known, the control scheme proposed in this paper is robust with respect to uncertainties in the model, see Section 6. Also observe that the term $R_{i}$ cannot be directly compensated by means of the control input $u_{i}$ due to the delay and the on-off nature of the control signal.

We also consider the following initial conditions for (2),

$$
u_{i}(t)=0, \quad x_{i}(t)=0, \quad t \in\left[t_{0}-\tau_{\max }, t_{0}\right],
$$

where $\tau_{\max }$ is the maximum delay in the model. Note that the initial time can be chosen as $t_{0}=0$.

Since we want (2) to model the input-output behaviour of a flow separation system, it must fulfil the following characteristics of physical meaning.

C1. According to the nature of the sensors, the output $x_{i}(t)=0$ describes the flow in a stable steady-state separated condition, and $x_{i}(t)>0$ describes a reduction in the flow separation (or re-attachment of the flow). [7] Thus:

- the parameters of the model must be such that the solutions of the system are nonnegative;

- for $u_{i} \equiv 0, i=1, \ldots, n$, the origin of the system is an asymptotically stable equilibrium point.

C2. The nature of the flow is such that, for any uniformly bounded input, the outputs of the system are uniformly bounded.

C3. According to the experimental settings considered in Feingesicht, [5] the actuators are on-off valves, hence the control inputs $u_{i}$ are restricted to take values in the set $\{0,1\}$.

We have assumed above that the parameters of the model are known; Nevertheless, the conditions on the parameters of (2) that ensure the fulfilment of $\mathrm{C} 1$ and $\mathrm{C} 2$ are given in Section 3. Of course, the controller designed in Section 4 accomplish C3.

The control objective is to design the feedback controllers $u_{i}$ to take the trajectories of (2) out of the origin, and to drive them to a reference point $x^{*}=\left[x_{1}^{*}, \ldots, x_{n}^{*}\right]^{\top}$. This represents a reduction of the flow separation to a desired level.

Note that the model is an input-output representation of the system and the inputs and outputs are related with the actuators and sensors, respectively. Hence, no observation scheme is required by the controller designed in Section 4. In this sense, the proposed

\footnotetext{
${ }^{2}$ The methodology developed in [5] can be used for parametric identification of (2).
} 
model adapts to the provided instrumentation of the system. Of course, it is expected that a higher number of sensors and actuators would help to improve the performance of the controlled system, however, the quantity and placing of the instrumentation is a topic that requires a deeper study, to be conducted in relation with fluid mechanics theory. Thus, it is not dealt with in this paper.

We assume that for each sensor the closest actuator is dominant, i.e., for each $x_{i}$ the input $u_{i}$ is more influential than the other inputs $u_{j}, j \neq i$. A consequence of this consideration is the size of the delays in the interconnection terms of each output dynamics, this is considered in the following assumption.

Assumption 1. All the delays in (2) are constant and known. Moreover, for $i, j, r=$ $1, \ldots, n, k=1, \ldots, N_{i}^{1}, s=1, \ldots, N_{i}^{2}, s^{\prime}=1, \ldots, N_{i}^{3}$, we assume that

$$
\bar{\tau}_{i}^{s}, \tau_{i j}^{k}, \bar{\tau}_{i j}^{s^{\prime}}, \varsigma_{i}^{s, r}, \varsigma_{i j}^{s^{\prime}, r}, \varsigma_{i j}>\varsigma_{i}>0 .
$$

Now, let us recall some details about the existence of solutions of (2).

Remark 2 (On the solutions). A function $x: \mathbb{R}_{\geq t_{0}-\tau_{\max }} \rightarrow \mathbb{R}^{n}$, that is locally absolutely continuous on $t \in\left[t_{0}, \infty\right)$, is called a solution of problem (2), (3) if it satisfies (2) for almost all $t \in\left[t_{0}, \infty\right)$, and (3) for all $t \leq t_{0}$, see e.g., page 100 in Kolmanovskii and Myshkis. [12] If $u:\left[t_{0}-\tau_{\max }, \infty\right) \rightarrow \mathbb{R}^{m}$ is a Lebesgue-measurable and locally essentially bounded function, then (2) satisfies the Carathéodory conditions. Hence, there exists a unique solution of the problem (2), (3), see e.g., page 100 in the book of Kolmanovskii and Myshkis, [12] or page 58 in the book of Hale and Verduyn Lunel. [10] In the feedback case the existence of solutions can be proven by the method of steps, see Section 4.3.

In fact, the controller designed in Section 4 guarantees that the control signal $u$ is a Lebesgue-measurable function of time, see Section 4.3 .

\section{$3 \quad$ Stability properties of the model}

In this section we give conditions on the parameters of (2) that guarantee the requirements in $\mathrm{C} 1$ and $\mathrm{C} 2$. First, let us rewrite (2) as follows

$$
\dot{x}_{i}(t)=\sum_{k} a_{i}^{k} x_{i}\left(t-\tau_{i}^{k}\right)+\sum_{s} \bar{\alpha}_{i}^{s}(t) x_{i}\left(t-\bar{\tau}_{i}^{s}\right)+b_{i} u_{i}\left(t-\varsigma_{i}\right)+R_{i}(t)
$$

where

$$
\bar{\alpha}_{i}^{s}(t):=u_{i}\left(t-\varsigma_{i}\right) \bar{a}_{i}^{s}+\sum_{r \neq i} u_{r}\left(t-\varsigma_{i}^{s, r}\right) \bar{a}_{i}^{s, r}
$$

Note that (4) can be considered as a linear time-delay system with time-varying coefficients, which is true even in the feedback case due to the delay in the input, see Section 4.3 . Moreover, according to the restrictions on the control inputs, the time-varying coefficients $\bar{\alpha}_{i}^{s}(t)$ can take only a finite number of different values. 


\subsection{Stability and positivity}

In this section, we use the concept of the Cauchy matrix to state the stability and positivity properties of (4). Such a concept is recalled in Appendix A.1.

Notation 1. We say that (4) is in its homogeneous form if:

- for Case 1, $b_{i}=0$ and $R_{i}(t) \equiv 0$ for all $i \in\{1, \ldots, n\}$;

- for Case 2, $b_{i}=b_{i j}=0$ for all $i, j \in\{1, \ldots, n\}$.

Definition 1 (Domoshnitsky and Fridman [4]). The homogeneous form of (4) is said to be:

- positive, if all the entries of its Cauchy matrix $C(t, \eta)$ are nonnegative in the triangle $0 \leq \eta \leq t<\infty$

- exponentially stable, if there exist $\beta_{1}, \beta_{2} \in \mathbb{R}_{>0}$ such that all the entries of its Cauchy matrix $C(t, \eta)$ satisfy

$$
\left|C_{i j}(t, \eta)\right| \leq \beta_{1} \exp \left(-\beta_{2}(t-\eta)\right), \quad i, j=1, \ldots, n, \quad 0 \leq \eta \leq t<\infty .
$$

We recall in the following two lemmas two procedures to verify positivity and exponential stability of (4). To this end, we need the following assumption.

Assumption 2. If $\bar{a}_{i}^{s} \neq 0$ and $\bar{a}_{i}^{s, r} \neq 0$, then $\operatorname{sign}\left(\bar{a}_{i}^{s}\right)=\operatorname{sign}\left(\bar{a}_{i}^{s, r}\right)$.

This assumption guarantees that the sign of each coefficient $\bar{\alpha}_{i}^{s}(t)$ does not change for all $t \in \mathbb{R}$. Observe that, according to the properties of $u$ (see Section 4.3) we have that $\bar{\alpha}_{i}^{s} \in L^{\infty}(\mathbb{R})$ for all $i=1, \ldots, n$ and all $s=1, \ldots, N_{i}^{2}$. Now, for each $i=1, \ldots, n$, define the following sets $N_{k}(i)=\left\{k: a_{i}^{k}<0\right\}, P_{k}(i)=\left\{k: a_{i}^{k}>0\right\}, Z_{s}(i)=\left\{s: \bar{\alpha}_{i}^{s}(t)=\right.$ $\left.0, \forall t \in \mathbb{R}_{\geq t_{0}}\right\}, N_{s}(i)=\left\{s: \bar{\alpha}_{i}^{s}(t) \leq 0, \forall t \in \mathbb{R}_{\geq t_{0}}\right\} \backslash Z_{s}(i), P_{s}(i)=\left\{s: \bar{\alpha}_{i}^{s}(t) \geq 0, \forall t \in\right.$ $\left.\mathbb{R}_{\geq t_{0}}\right\} \backslash Z_{s}(i)$.

Lemma 1 (Domoshnitsky and Fridman [4]). Under Assumption 2, let the following conditions be fulfilled.

1. For every $i=1, \ldots, n$, at least one of the conditions 1(a) or 1(b) is fulfilled:

$$
\begin{aligned}
\text { 1(a) } \min _{k \in N_{k}(i), s \in N_{s}(i)}\left\{\tau_{i}^{k}, \bar{\tau}_{i}^{s}\right\} \geq \max _{k \in P_{k}(i), s \in P_{s}(i)}\left\{\tau_{i}^{k}, \bar{\tau}_{i}^{s}\right\}, \\
\quad-\sum_{k \in N_{k}(i)} a_{i}^{k}-\sum_{s \in N_{s}(i)} \bar{\alpha}_{i}^{s}(t) \geq \frac{1}{e}\left(\sum_{k \in P_{k}(i)} a_{i}^{k}+\sum_{s \in P_{s}(i)} \bar{\alpha}_{i}^{s}(t)\right), \quad \forall t \in \mathbb{R}_{\geq t_{0}},
\end{aligned}
$$

and for $\tau_{i}^{+}=\max _{k \in N_{k}(i), s \in N_{s}(i)}\left\{\tau_{i}^{k}, \bar{\tau}_{i}^{s}\right\}$, and all $t \in \mathbb{R}_{>t_{0}}$,

$$
\int_{t-\tau_{i}^{+}}^{t}\left[-\sum_{k \in N_{k}(i)} a_{i}^{k}-\sum_{s \in N_{s}(i)} \bar{\alpha}_{i}^{s}(\nu)-\frac{1}{e}\left(\sum_{k \in P_{k}(i)} a_{i}^{k}+\sum_{s \in P_{s}(i)} \bar{\alpha}_{i}^{s}(\nu)\right)\right] \mathrm{d} \nu \leq \frac{1}{e}
$$


1(b) $\max _{k \in N_{k}(i), s \in N_{s}(i)}\left\{\tau_{i}^{k}, \bar{\tau}_{i}^{s}\right\} \leq \min _{k \in P_{k}(i), s \in P_{s}(i)}\left\{\tau_{i}^{k}, \bar{\tau}_{i}^{s}\right\}$,

$$
\begin{gathered}
-\sum_{k \in N_{k}(i)} a_{i}^{k}-\sum_{s \in N_{s}(i)} \bar{\alpha}_{i}^{s}(t) \geq \sum_{k \in P_{k}(i)} a_{i}^{k}+\sum_{s \in P_{s}(i)} \bar{\alpha}_{i}^{s}(t), \quad \forall t \in \mathbb{R}_{\geq t_{0}}, \\
\int_{t-\tau_{i}^{+}}^{t}\left[-\sum_{k \in N_{k}(i)} a_{i}^{k}-\sum_{s \in N_{s}(i)} \bar{\alpha}_{i}^{s}(\nu)-\sum_{k \in P_{k}(i)} a_{i}^{k}-\sum_{s \in P_{s}(i)} \bar{\alpha}_{i}^{s}(t)\right] \mathrm{d} \nu \leq \frac{1}{e}, t \in \mathbb{R}_{\geq t_{0}}, \\
\text { where } \tau_{i}^{+}=\max _{k \in P_{k}(i), s \in P_{s}(i)}\left\{\tau_{i}^{k}, \bar{\tau}_{i}^{s}\right\}, \text { and } \\
\quad \int_{t}^{t+\delta_{\tau}}\left(-\sum_{k \in N_{k}(i)} a_{i}^{k}-\sum_{s \in N_{s}(i)} \bar{\alpha}_{i}^{s}(\nu)\right) \mathrm{d} \nu \leq \frac{1}{e}, t \in \mathbb{R}_{\geq t_{0}}, \\
\text { where } \delta_{\tau}=\max _{k \in P_{k}(i), s \in P_{s}(i)}\left\{\tau_{i}^{k}, \bar{\tau}_{i}^{s}\right\}-\min _{k \in N_{k}(i), s \in N_{s}(i)}\left\{\tau_{i}^{k}, \bar{\tau}_{i}^{s}\right\} .
\end{gathered}
$$

2. The coeficients $a_{i j}^{k}, \bar{a}_{i j}^{s^{\prime}, r} \geq 0$, for $k=1, \ldots, N_{i}^{1}, s^{\prime}=1, \ldots, N_{i}^{3}, i, j, r=1, \ldots, n$.

Then the homogeneous form of (4) is positive.

Lemma 2 (Domoshnitsky and Fridman [4]). Under Assumption 2, if the following conditions (1) and (2) are true:

(1) the condition (1) of Lemma 1 is fulfilled;

(2) there exist positive numbers $z_{1}, \ldots, z_{n}$ such that, for all $t \in \mathbb{R}_{\geq t_{0}}$,

$$
-\left(\sum_{k} a_{i}^{k}+\sum_{s} \bar{\alpha}_{i}^{s}(t)\right) z_{i}-\sum_{j \neq i}\left(\sum_{k} a_{i j}^{k}+\sum_{s^{\prime}, r} u_{r}\left(t-\varsigma_{i j}^{s^{\prime}, r}\right) \bar{a}_{i j}^{s^{\prime}, r}\right) z_{j} \geq 1,
$$

then: a) the homogeneous form of (4) is exponentially stable; b) the following estimates hold

$$
\underset{t \in \mathbb{R}_{\geq t_{0}}}{\operatorname{esssup}} \int_{t_{0}}^{t} \sum_{j}\left|C_{i, j}(t, \eta)\right| \mathrm{d} \eta \leq z_{i}
$$

Remark 3. If the hypothesis number 2 in Lemma 1 and condition (5) hold, then $\operatorname{ess}_{\sup }\left(\sum_{k} a_{i}^{k}+\sum_{s} \bar{\alpha}_{i}^{s}(t)\right)<0$ for each $i=1, \ldots, n$.

Now, we can state the following result on the nonnegative solutions of (4).

Lemma 3. Consider (4). Suppose that, for each $i \in\{1, \ldots, n\}, u_{i} \in L^{\infty}\left(\mathbb{R}_{\geq 0}\right), u_{i}(t) \in$ $\{0,1\}$ for all $t \in \mathbb{R}_{\geq 0}$, and

- for Case $\mathbf{1}, b_{i} \in \mathbb{R}_{\geq 0}, R_{i}(t) \geq 0$ for all $t \in \mathbb{R}_{\geq 0}$ for all $i \in\{1, \ldots, n\}$,

- for Case 2, $b_{i}, b_{i j} \in \mathbb{R}_{\geq 0}$.

If the homogeneous form of (4) is positive, then the solutions of the problem (4), (3) are such that $x_{i}(t) \geq 0$ for all $t \in \mathbb{R}_{\geq t_{0}}$ for all $i \in\{1, \ldots, n\}$.

Proof. The result is straightforward by using the solution representation with the Cauchy matrix (see Lemma 5 in Appendix A.1. 


\subsection{Boundedness}

Lemma 2 gives conditions to guarantee exponential stability of (4) in its homogeneous form. This guarantees boundedness of the trajectories in the presence of bounded inputs, and allows us to compute a quantitative bound for the trajectories of the system and their derivatives.

Lemma 4. If (4), with the initial conditions (3), satisfies Lemmas 2 and 3, then

$$
\left\|x_{i}(t)\right\|_{L^{\infty}} \leq z_{i} \max _{j^{\prime} \in\{1, \ldots, n\}} \bar{B}_{j^{\prime}}
$$

with $z_{i}$ as defined in (5), and

- for Case 1, $\bar{B}_{j^{\prime}}:=b_{j^{\prime}}+\left\|R_{j^{\prime}}(t)\right\|_{L^{\infty}}$;

- for Case $2, \bar{B}_{j^{\prime}}:=b_{j^{\prime}}+\sum_{j \neq j^{\prime}} b_{j^{\prime} j}$.

Moreover, if $\operatorname{ess}^{\sup _{t \in \mathbb{R}}}\left(\sum_{k}\left|a_{i}^{k}\right| \tau_{i}^{k}+\sum_{s}\left|\bar{\alpha}_{i}^{s}(t)\right| \bar{\tau}_{i}^{s}\right)<1$, then

$$
\left\|\dot{x}_{i}(t)\right\|_{L^{\infty}} \leq \frac{\max \left(\gamma_{i, 1}, \gamma_{i, 2}\right)}{1-\operatorname{ess}_{\sup _{t \in \mathbb{R}}}\left(\sum_{k}\left|a_{i}^{k}\right| \tau_{i}^{k}+\sum_{s}\left|\bar{\alpha}_{i}^{s}(t)\right| \bar{\tau}_{i}^{s}\right)}
$$

with $\gamma_{i, 2}=\left\|x_{i}(t)\right\|_{L^{\infty}} \operatorname{ess}_{\sup _{t \in \mathbb{R}}}\left(-\sum_{k} a_{i}^{k}-\sum_{s} \bar{\alpha}_{i}^{s}(t)\right)$, and

- for Case 1, $\gamma_{i, 1}=\bar{B}_{i}$;

- for Case 2, $\gamma_{i, 1}=\sum_{j \neq i}\left(\sum_{k} a_{i j}^{k}+\sum_{s^{\prime}, r} \bar{a}_{i j}^{s^{\prime}, r}\right)\left\|x_{j}(t)\right\|_{L^{\infty}}+\bar{B}_{i}$.

Proof. We give the proof for $R_{i}$ as in Case 2, the proof for Case $\mathbf{1}$ is analogous. Define $B_{i}(t):=b_{i} u_{i}\left(t-\varsigma_{i}\right)+\sum_{j \neq i} b_{i j} u_{j}\left(t-\varsigma_{i j}\right)$. According to Lemma 5 (see Appendix A.1), the solution of the problem (4), (3) can be written as follows

$$
x_{i}(t)=\int_{t_{0}}^{t} \sum_{j} C_{i, j}(t, \eta) B_{j}(\eta) \mathrm{d} \eta
$$

where $C(t, \eta)$ is the Cauchy matrix of (4). Hence, we have that

$$
\left|x_{i}(t)\right| \leq \int_{t_{0}}^{t} \sum_{j}\left|C_{i, j}(t, \eta)\right|\left\|B_{j}(t)\right\|_{L^{\infty}} \mathrm{d} \eta \leq \int_{t_{0}}^{t} \sum_{j}\left|C_{i, j}(t, \eta)\right| \mathrm{d} \eta \max _{j^{\prime}} \bar{B}_{j^{\prime}} \leq z_{i} \max _{j^{\prime}} \bar{B}_{j^{\prime}}
$$

thus, $\left\|x_{i}(t)\right\|_{L^{\infty}} \leq z_{i} \max _{j^{\prime}} \bar{B}_{j^{\prime}}$.

The proof for the second part of the lemma is divided in two cases. First, suppose that $\dot{x}_{i}(t)>0$. From (4) we obtain the inequality

$$
\dot{x}_{i}(t) \leq \sum_{k} a_{i}^{k} x_{i}\left(t-\tau_{i}^{k}\right)+\sum_{s} \bar{\alpha}_{i}^{s}(t) x_{i}\left(t-\bar{\tau}_{i}^{s}\right)+\gamma_{i, 1}
$$


which can be rewritten as follows

$\dot{x}_{i}(t) \leq\left(\sum_{k} a_{i}^{k}+\sum_{s} \bar{\alpha}_{i}^{s}(t)\right) x_{i}(t)-\sum_{k} a_{i}^{k} \int_{t-\tau_{i}^{k}}^{t} \dot{x}_{i}(\nu) \mathrm{d} \nu-\sum_{s} \bar{\alpha}_{i}^{s}(t) \int_{t-\bar{\tau}_{i}^{s}}^{t} \dot{x}_{i}(\nu) \mathrm{d} \nu+\gamma_{i, 1}$,

thus, taking into account Remark 3, we find the bound

$$
\left|\dot{x}_{i}(t)\right| \leq \underset{t}{\operatorname{ess} \sup }\left(\sum_{k}\left|a_{i}^{k}\right| \tau_{i}^{k}+\sum_{s}\left|\bar{\alpha}_{i}^{s}(t)\right| \bar{\tau}_{i}^{s}\right)\left\|\dot{x}_{i}(t)\right\|_{L^{\infty}}+\gamma_{i, 1}
$$

Now, suppose that $\dot{x}(t)<0$. From (4) we have that

$$
-\dot{x}_{i}(t) \leq-\sum_{k} a_{i}^{k} x_{i}\left(t-\tau_{i}^{k}\right)-\sum_{s} \bar{\alpha}_{i}^{s}(t) x_{i}\left(t-\bar{\tau}_{i}^{s}\right) .
$$

Since this inequality can be rewritten as

$$
-\dot{x}_{i}(t) \leq-\left(\sum_{k} a_{i}^{k}+\sum_{s} \bar{\alpha}_{i}^{s}(t)\right) x_{i}(t)+\sum_{k} a_{i}^{k} \int_{t-\tau_{i}^{k}}^{t} \dot{x}_{i}(\nu) \mathrm{d} \nu+\sum_{s} \bar{\alpha}_{i}^{s}(t) \int_{t-\bar{\tau}_{i}^{s}}^{t} \dot{x}_{i}(\nu) \mathrm{d} \nu
$$

we can obtain the bound

$$
\begin{aligned}
\left|\dot{x}_{i}(t)\right| \leq & \operatorname{ess}_{t} \sup \left(-\sum_{k} a_{i}^{k}-\sum_{s} \bar{\alpha}_{i}^{s}(t)\right)\left\|x_{i}(t)\right\|_{L^{\infty}}+ \\
& \quad \operatorname{essup}_{t}\left(\sum_{k}\left|a_{i}^{k}\right| \tau_{i}^{k}+\sum_{s}\left|\bar{\alpha}_{i}^{s}(t)\right| \bar{\tau}_{i}^{s}\right)\left\|\dot{x}_{i}(t)\right\|_{L^{\infty}} .
\end{aligned}
$$

The result follows from the comparison of (8) and (9), and by the assumption $\operatorname{essup}_{t \in \mathbb{R}}\left(\sum_{k}\left|a_{i}^{k}\right| \tau_{i}^{k}+\sum_{s}\left|\bar{\alpha}_{i}^{s}(t)\right| \bar{\tau}_{i}^{s}\right)<1$.

\subsection{Asymptotic bounds of $x_{i}(t)$}

In Section 3.2 we have verified the boundedness of the trajectories of $(2)$ for any bounded input. In this section we investigate some asymptotic bounds (or bounds in steady-state) of each subsystem in (4) by considering constant inputs and regarding the interconnection terms as disturbances. For such an analysis we need to define the following coefficients from (2).

- Define $c_{i}^{s}=-\sum_{r \in \Omega_{i}^{s}} \bar{a}_{i}^{s, r}, \Omega_{i}^{s}=\left\{r \in\{1, \ldots, n\}: r \neq i, \bar{a}_{i}^{s, r}<0\right\}$. If for some $s$ the set $\Omega_{i}^{s}$ is empty, then define $c_{i}^{s}=0$.

- Define $\bar{c}_{i}^{s}=\sum_{r \in \bar{\Omega}_{i}^{s}} \bar{a}_{i}^{s, r}, \bar{\Omega}_{i}^{s}=\left\{r \in\{1, \ldots, n\}: r \neq i, \bar{a}_{i}^{s, r}>0\right\}$. If for some $s$ the set $\bar{\Omega}_{i}^{s}$ is empty, then define $\bar{c}_{i}^{s}=0$.

Claim 1. Assume that (4) satisfies all the conditions of Lemma 4, and $u_{i}(t) \equiv 1$. Then $x_{i}(t) \geq \bar{y}_{i}$ if $t \rightarrow \infty$, where

$$
\bar{y}_{i}=b_{i}\left(-\sum_{k} a_{i}^{k}-\sum_{s}\left(\bar{a}_{i}^{s}-c_{i}^{s}\right)\right)^{-1} .
$$


Proof. Since (4) satisfies all the conditions of Lemma $4, R_{i}(t) \geq 0$ for all $t \in \mathbb{R}$. Also observe that the term $R_{i}$ does not depend on $x_{i}$. Thus, for $u_{i}(t) \equiv 1$ and $t \geq t_{0}+\varsigma_{i}$, we have that (4) is given by

$$
\begin{aligned}
\dot{x}_{i}(t)= & \sum_{k} a_{i}^{k} x_{i}\left(t-\tau_{i}^{k}\right)+\sum_{s} \bar{a}_{i}^{s} x_{i}\left(t-\bar{\tau}_{i}^{s}\right)+b_{i}+ \\
& \sum_{s, r \neq i} u_{r}\left(t-\varsigma_{i}^{s, r}\right) \bar{a}_{i}^{s, r} x_{i}\left(t-\bar{\tau}_{i}^{s}\right)+R_{i}(t) .
\end{aligned}
$$

Note that the right-hand side of (11) depends on $u_{r}$ ( with $r \neq i$ ) and on $R_{i}$. Since $x_{i}$ is nonnegative, we have that $\sum_{s, r \neq i} u_{r}\left(t-\varsigma_{i}^{s, r}\right) \bar{a}_{i}^{s, r} x_{i}\left(t-\bar{\tau}_{i}^{s}\right) \geq-\sum_{s} c_{i}^{s} x_{i}\left(t-\bar{\tau}_{i}^{s}\right)$. Moreover, $R_{i}(t) \geq 0$ for all $t \in \mathbb{R}$. Therefore, the case of slowest growing rate of $x_{i}$ in (11) occurs if

$$
\dot{x}_{i}(t)=\sum_{k} a_{i}^{k} x_{i}\left(t-\tau_{i}^{k}\right)+\sum_{s}\left(\bar{a}_{i}^{s}-c_{i}^{s}\right) x_{i}\left(t-\bar{\tau}_{i}^{s}\right)+b_{i} .
$$

Observe that no assumption of lemmas 3 and 4 has been modified, thus, the homogeneous form of $(12)$ is positive and exponentially stable. Hence, Lemma 6 (see Appendix A.1 asserts that the solution of (12) is such that if $t \rightarrow \infty$, then $x_{i}(t) \rightarrow \bar{y}_{i}$.

Claim 2. Assume that (4) satisfies all the conditions of Lemma 4, and $u_{i}(t) \equiv 0$. Then $x_{i}(t) \leq \underline{y}_{i}$ if $t \rightarrow \infty$, where

$$
\underline{y}_{i}=\left\|R_{i}(t)\right\|_{L^{\infty}}\left(-\sum_{k} a_{i}^{k}-\sum_{s} \bar{c}_{i}^{s}\right)^{-1} .
$$

Proof. For $u_{i}(t) \equiv 0$ and $t \geq t_{0}+\varsigma_{i}$, (4) is as follows

$$
\dot{x}_{i}(t)=\sum_{k} a_{i}^{k} x_{i}\left(t-\tau_{i}^{k}\right)+\sum_{s, r \neq i} u_{r}\left(t-\varsigma_{i}^{s, r}\right) \bar{a}_{i}^{s, r} x_{i}\left(t-\bar{\tau}_{i}^{s}\right)+R_{i}(t) .
$$

Since $x_{i}$ is nonnegative, we have that $\sum_{s, r \neq i} u_{r}\left(t-\varsigma_{i}^{s, r}\right) \bar{a}_{i}^{s, r} x_{i}\left(t-\bar{\tau}_{i}^{s}\right) \leq \sum_{s} \bar{c}_{i}^{s} x_{i}\left(t-\bar{\tau}_{i}^{s}\right)$. Thus, the case of fastest growing rate of $x_{i}$ in 14 occurs if

$$
\dot{x}_{i}(t)=\sum_{k} a_{i}^{k} x_{i}\left(t-\tau_{i}^{k}\right)+\sum_{s, r \neq i} \bar{c}_{i}^{s} x_{i}\left(t-\bar{\tau}_{i}^{s}\right)+\left\|R_{i}(t)\right\|_{L^{\infty}} .
$$

Again, since the assumptions of lemmas 3 and 4 have not been modified, the homogeneous form of 15 is positive and exponentially stable. Hence, and according to Lemma 6 (see Appendix A.1), the solution of (14) is such that if $t \rightarrow \infty$, then $x_{i}(t) \rightarrow \underline{y}_{i}$.

Note that, from Remark 3, we have that $\underline{y}_{i}, \bar{y}_{i} \geq 0$.

\section{Sliding mode controller}

In this section we provide a control scheme for (2) that accomplish the requirement in C3 (see Section 2). 


\subsection{On the reference points}

Since the control signals and the solutions of (2) are bounded, the reference points $x_{i}^{*}$ cannot be arbitrarily chosen. Thus, an admissible range for them must be specified. In this section, we give a procedure to compute a range of reference points, which is suitable for the controller to be designed below.

1. Consider (2) and the constants $c_{i}^{s}, \bar{c}_{i}^{s}$ defined in Section 3.3. Define the constants $E_{i}=\sum_{k}\left|a_{i}^{k}\right| \tau_{i}^{k}+\sum_{s}\left|\bar{a}_{i}^{s}\right| \bar{\tau}_{i}^{s}$. Choose $D_{i} \in \mathbb{R}_{>0}$ such that $\left\|\dot{x}_{i}(t)\right\|_{L^{\infty}} \leq D_{i}$, and define

$$
\bar{x}_{i}^{*}=\frac{b_{i}-\left[E_{i}+\sum_{s} c_{i}^{s}\left(\bar{\tau}_{i}^{s}-\varsigma_{i}\right)\right] D_{i}}{-\sum_{k} a_{i}^{k}-\sum_{s}\left(\bar{a}_{i}^{s}-c_{i}^{s}\right)} .
$$

2. Choose $\bar{R}_{i} \in \mathbb{R}_{\geq 0}$ such that $\left\|R_{i}(t)\right\|_{L^{\infty}} \leq \bar{R}_{i}$, and define $\check{x}_{i}=\bar{R}_{i}\left(-\sum_{k} a_{i}^{k}-\sum_{s} \bar{c}_{i}^{s}\right)^{-1}$. Define the constants $\bar{E}_{i}=\sum_{k}\left|a_{i}^{k}\right| \tau_{i}^{k}+\sum_{s}\left|\bar{a}_{i}^{s}\right| \varsigma_{i}$,

$$
x_{i}^{d}=\frac{\left[\bar{E}_{i}+\sum_{s} \bar{c}_{i}^{s}\left(\bar{\tau}_{i}^{s}-\varsigma_{i}\right)\right] D_{i}+\bar{R}_{i}}{-\sum_{k} a_{i}^{k}-\sum_{s} \bar{c}_{i}^{s}}, \quad \tilde{x}_{i}=\frac{x_{i}^{d}+\varsigma_{i}\left(b_{i}+\bar{R}_{i}\right)}{1-\varsigma_{i}\left(\sum_{k} a_{i}^{k}+\sum_{s}\left(\bar{a}_{i}^{s}+\bar{c}_{i}^{s}\right)\right)},
$$

and

$$
\underline{x}_{i}^{*}=\max \left(\check{x}_{i}, \tilde{x}_{i}\right) \text {. }
$$

Thus, a range of reference points is given by $\left(\underline{x}_{i}^{*}, \bar{x}_{i}^{*}\right)$ if the following inequality

$$
\bar{x}_{i}^{*}>\underline{x}_{i}^{*},
$$

holds. The following are important remarks on this procedure:

- a necessary condition to fulfil $(19)$ is that $b_{i}>\left[E_{i}+\sum_{s} c_{i}^{s}\left(\bar{\tau}_{i}^{s}-\varsigma_{i}\right)\right] D_{i}$;

- an immediate option for $D_{i}$ is the bound given in (7). For $R_{i}$ as in Case 2, an option for the bound $\bar{R}_{i}$ is obtained by considering the estimate $\left\|R_{i}(t)\right\|_{L^{\infty}} \leq$ $\sum_{j \neq i}\left(\sum_{k} a_{i j}^{k}+\sum_{s^{\prime}, r} \bar{a}_{i j}^{s^{\prime}, r}\right)\left\|x_{j}(t)\right\|_{L^{\infty}}+\sum_{j \neq i} b_{i j}$, and the bounds for $\left\|x_{j}(t)\right\|_{L^{\infty}}$ given in 6 ;

- it is easy to see that $\left(\underline{x}_{i}^{*}, \bar{x}_{i}^{*}\right) \subset\left(\underline{y}_{i}, \bar{y}_{i}\right)$.

\subsection{Sliding Mode controller}

To solve the problem stated in Section 2 we propose a sliding mode controller. The control law is as follows

$$
u_{i}(t)= \begin{cases}1, & \sigma_{i}(t)<0 \\ 0, & \sigma_{i}(t)>0\end{cases}
$$

where the sliding variable $\sigma_{i}$ is given by

$$
\sigma_{i}(t)=x_{i}(t)+T_{i}(t)-\sigma_{i}^{*}
$$


where $\sigma_{i}^{*}:=\left(1-\varsigma_{i} \sum_{k} a_{i}^{k}-\varsigma_{i} \sum_{s} \bar{a}_{i}^{s}\right) x_{i}^{*}$, and

$$
\begin{aligned}
T_{i}(t):= & \int_{t-\varsigma_{i}}^{t}\left[\left(u_{i}(\eta)-1\right) \sum_{s} \bar{a}_{i}^{s} x_{i}\left(\eta-\bar{\tau}_{i}^{s}+\varsigma_{i}\right)+b_{i} u_{i}(\eta)+\right. \\
& \left.\sum_{s, r \neq i} u_{r}\left(\eta-\varsigma_{i}^{s, r}+\varsigma_{i}\right) \bar{a}_{i}^{s, r} x_{i}\left(\eta-\bar{\tau}_{i}^{s}+\varsigma_{i}\right)+R_{i}\left(\eta+\varsigma_{i}\right)\right] \mathrm{d} \eta .
\end{aligned}
$$

To study the stability features in sliding regime we need the following. For $i=1, \ldots, n$, define the functions $K_{i}: \mathbb{R}_{\geq 0} \rightarrow \mathbb{R}$ given by

$$
K_{i}(r)=\sum_{k} A_{i}^{k}(r)+\sum_{s} \bar{A}_{i}^{s}(r)
$$

where

$$
A_{i}^{k}(r):=\left\{\begin{array}{cc}
-a_{i}^{k}, & r \in\left[\tau_{i}^{k}, \tau_{i}^{k}+\varsigma_{i}\right] \\
0, & r \notin\left[\tau_{i}^{k}, \tau_{i}^{k}+\varsigma_{i}\right]
\end{array} \quad, \quad \bar{A}_{i}^{s}(r):=\left\{\begin{array}{cc}
-\bar{a}_{i}^{s}, & r \in\left[\bar{\tau}_{i}^{s}, \bar{\tau}_{i}^{s}+\varsigma_{i}\right] \\
0, & r \notin\left[\bar{\tau}_{i}^{s}, \bar{\tau}_{i}^{s}+\varsigma_{i}\right]
\end{array} .\right.\right.
$$

Now we are ready to state the main result of this section.

Theorem 1. Suppose that (2), with initial conditions (3), satisfies Lemma 4. If (18) and (16) are such that (19) holds, then for any $x_{i}^{*} \in\left(\underline{x}_{i}^{*}, \bar{x}_{i}^{*}\right)$, the following is true for the closed-loop (2), 20):

- a sliding motion is established in a finite-time on $\sigma_{i}=0$;

- if additionally, the condition

$$
\left\|K_{i}(r)\right\|_{L^{1}\left(\mathbb{R}_{\geq 0}\right)}<1
$$

holds, then in the sliding motion, $x_{i}$ converges exponentially to $x_{i}^{*}$.

The proof of this theorem is given in Section 5 .

Remark 4. Let us comment some important properties of the controller.

- Observe that in Theorem 1, the sliding motion and the exponential convergence of $x_{i}$ to $x_{i}^{*}$ are guaranteed independently of the steady-state behaviour of $x_{j}$ for all $j \neq i$. This reflects the decentralized-like feature of this sliding mode controller.

- The controller has the ability to compensate the interconnection terms in the sliding motion not by asking a high gain of the control input, but by a suitable design of the sliding variable.

- The computational cost required by the control law is very low. Note that for a digital implementation the integral term can be computed as simple recursive sum. 


\subsection{On the closed-loop solutions}

In Remark 2 we have established a framework to guarantee the existence and uniqueness of solutions of (2). In this section we verify that such a framework is still valid for the closed loop system.

The time derivative of the sliding variable $\sigma_{i}$ is given by

$$
\begin{gathered}
\dot{\sigma}_{i}(t)=\sum_{k} a_{i}^{k} x_{i}\left(t-\tau_{i}^{k}\right)+\sum_{s} \bar{a}_{i}^{s} x_{i}\left(t-\bar{\tau}_{i}^{s}\right)-\left(1-u_{i}(t)\right) \sum_{s} \bar{a}_{i}^{s} x_{i}\left(t-\bar{\tau}_{i}^{s}+\varsigma_{i}\right)+ \\
b_{i} u_{i}(t)+\sum_{s, r \neq i} u_{r}\left(t-\varsigma_{i}^{s, r}+\varsigma_{i}\right) \bar{a}_{i}^{s, r} x_{i}\left(t-\bar{\tau}_{i}^{s}+\varsigma_{i}\right)+R_{i}\left(t+\varsigma_{i}\right) .
\end{gathered}
$$

Note that the right-hand side of (24) depends on $x$, and in turn, the right-hand side of (2) in closed-loop with (20) depends on $\sigma$. Moreover, the right-hand side of (24) is discontinuous in $\sigma$. Thus, in general, (24), (2) is a system of discontinuous functional differential equations. Nevertheless, the existence and uniqueness of solutions of such a system can still be guaranteed by means of standard definitions, as we explain in the following points.

- The solution of the problem (2), (3) can be obtained by means of the method of steps (see e.g., page 89 in the book of Kolmanovskii and Myshkis [12]), i.e. by solving (2) for $t$ in the intervals $\left[t_{0}, t_{0}+\tau_{\min }\right],\left[t_{0}+\tau_{\min }, t_{0}+2 \tau_{\min }\right]$, and so on, where $\tau_{\min }$ denotes the minimum of all the non-zero delays in (2).

- Consider the first interval $\left[t_{0}, t_{0}+\tau_{\min }\right]$, and note that for all $t$ in this interval, due to the input delay, (2) does not depend on $\sigma(t)$ but on $\sigma(t-\varsigma)$, which is an already known function. Thus, for each $t \in\left[t_{0}, t_{0}+\tau_{\min }\right],(2)$ is independent of the evolution of (24). Hence, the definition of solution given in Remark 2 is still valid for (2).

- Analogously, in the same time interval (i.e. for all $t \in\left[t_{0}, t_{0}+\tau_{\min }\right]$ ), (24) does not depend on the evolution of (2). Hence, (24) can be seen as a nonautonomous differential equation with discontinuous right-hand side, therefore, we can use the definition of solutions $3^{3}$ given in page 50 of the book of Filippov. [8]

- For the subsequent time intervals, the same argumentation is used to obtain the whole solution of the closed-loop system.

We claimed at the end of Section 2 that the control signal is a Lebesgue-measurable function of $t$. To verify this, we only have to note that since $\sigma_{i}$ and $x_{i}$ are absolutely continuous functions of $t$, then from the integral representation of $\sigma_{i}$ (given by (21)) we have that $u_{i}$ is necessarily Lebesgue-measurable in $t$ (see e.g. [17, p. 109-110]).

\footnotetext{
${ }^{3}$ For the particular case of (24), the three methods given in pages 50-56 in the book of Filippov [8] to construct the differential inclusion coincide, see also the work of Polyakov and Fridman. 16]
} 


\section{Proof of Theorem 1}

The proof is divided in three main parts, first we verify that the solutions of the system reach the condition $\sigma_{i}(t)=0$ in finite time. In the second part, the establishment of the sliding regime is confirmed. The last part consists in analysing the behaviour of the solution of the system during the sliding motion.

\section{$5.1 \quad$ Reaching analysis}

From Remark 3 it is clear that each $\sigma_{i}^{*}$ is strictly positive, thus, for the initial conditions (3) we have that $\sigma_{i}\left(t_{0}\right)<0$. If for $t=t_{0}+\varsigma_{i}, \sigma_{i}(t)$ is still negative, then $u_{i}(t)=1$, and

$$
\sigma_{i}(t)=x_{i}(t)+b_{i} \varsigma_{i}-\sigma_{i}^{*}+\int_{t-\varsigma_{i}}^{t}\left(\sum_{s, r \neq i} u_{r}\left(\eta-\varsigma_{i}^{s, r}+\varsigma_{i}\right) \bar{a}_{i}^{s, r} x_{i}\left(\eta-\bar{\tau}_{i}^{s}+\varsigma_{i}\right)+R_{i}\left(\eta+\varsigma_{i}\right)\right) \mathrm{d} \eta,
$$

for all $t \geq t_{0}+\varsigma_{i}$ such that $\sigma_{i}(t)<0$. Observe that, since $R_{i}(t) \geq 0$ for all $t \in \mathbb{R}_{\geq t_{0}}$,

$$
\sigma_{i}(t) \geq x_{i}(t)+\varsigma_{i} b_{i}-\sigma_{i}^{*}-\int_{t-\varsigma_{i}}^{t} \sum_{s} c_{i}^{s} x_{i}\left(\eta-\bar{\tau}_{i}^{s}+\varsigma_{i}\right) \mathrm{d} \eta,
$$

where the coefficients $c_{i}^{s}$ are as defined in Section 3.3. Since $x_{i}^{*}<\bar{y}_{i}$ (see Section 4.1 and Section 3.3), Claim 1 ensures the existence of $t_{1}$ such that $x_{i}\left(t_{1}\right)=x_{i}^{*}$. Therefore, from (25) we can see that

$$
\sigma_{i}\left(t_{1}\right) \geq x_{i}^{*}+\varsigma_{i} b_{i}-\sigma_{i}^{*}-\varsigma_{i} \sum_{s} c_{i}^{s} x_{i}^{*} .
$$

Now, from 10], $b_{i}=\bar{y}_{i}\left(-\sum_{k} a_{i}^{k}-\sum_{s}\left(\bar{a}_{i}^{s}-c_{i}^{s}\right)\right)$, thus (by considering the value of $\sigma_{i}^{*}$ )

$$
\begin{aligned}
\sigma_{i}\left(t_{1}\right) & \geq \varsigma_{i}\left(-\sum_{k} a_{i}^{k}-\sum_{s}\left(\bar{a}_{i}^{s}-c_{i}^{s}\right)\right) \bar{y}_{i}-\varsigma_{i}\left(-\sum_{k} a_{i}^{k}-\sum_{s} \bar{a}_{i}^{s}\right) x_{i}^{*}-\varsigma_{i} \sum_{s} c_{i}^{s} x_{i}^{*}, \\
& \geq \varsigma_{i}\left(-\sum_{k} a_{i}^{k}-\sum_{s} \bar{a}_{i}^{s}\right)\left(\bar{y}_{i}-x_{i}^{*}\right)+\varsigma_{i}\left(\bar{y}_{i}-x_{i}^{*}\right) \sum_{s} c_{i}^{s} .
\end{aligned}
$$

Since $x_{i}^{*}<\bar{y}_{i}$ and $\sum_{k} a_{i}^{k}+\sum_{s} \bar{a}_{i}^{s}<0$ (see Remark 3), we have that $\sigma_{i}\left(t_{1}\right) \geq 0$. This ensures the existence of $t_{i}^{*}$ such that $\sigma_{i}\left(t_{i}^{*}\right)=0$.

\subsection{Existence of the sliding mode}

We have proven that the sliding surface is reached, but now, we verify the existence of the sliding motion in the following two points.

1. Once the sliding surface is reached, i.e $\sigma_{i}(t)=0, u_{i}$ switches from $u_{i}(t)=1$ to $u_{i}(t)=0$, thus the dynamics of the sliding variable (given by (24)) becomes

$$
\begin{aligned}
\dot{\sigma}_{i}(t)= & \sum_{k} a_{i}^{k} x_{i}\left(t-\tau_{i}^{k}\right)+\sum_{s} \bar{a}_{i}^{s} x_{i}\left(t-\bar{\tau}_{i}^{s}\right)-\sum_{s} \bar{a}_{i}^{s} x_{i}\left(t-\bar{\tau}_{i}^{s}+\varsigma_{i}\right)+ \\
& \sum_{s, r \neq i} u_{r}\left(t-\varsigma_{i}^{s, r}+\varsigma_{i}\right) \bar{a}_{i}^{s, r} x_{i}\left(t-\bar{\tau}_{i}^{s}+\varsigma_{i}\right)+R_{i}\left(t+\varsigma_{i}\right) .
\end{aligned}
$$


We need to verify that, at the reaching instant, the switching of $u_{i}$ generates the condition $\dot{\sigma}_{i}(t)<0$. First note that

$$
\begin{aligned}
\dot{\sigma}_{i}(t) \leq & \sum_{k} a_{i}^{k} x_{i}\left(t-\tau_{i}^{k}\right)+\sum_{s} \bar{a}_{i}^{s} x_{i}\left(t-\bar{\tau}_{i}^{s}\right)-\sum_{s} \bar{a}_{i}^{s} x_{i}\left(t-\bar{\tau}_{i}^{s}+\varsigma_{i}\right)+ \\
& \sum_{s} \bar{c}_{i}^{s} x_{i}\left(t-\bar{\tau}_{i}^{s}+\varsigma_{i}\right)+R_{i}\left(t+\varsigma_{i}\right),
\end{aligned}
$$

where the coefficients $\bar{c}_{i}^{s}$ are as defined in Section 3.3. Note that this inequality can be rewritten as follows

$$
\begin{aligned}
\dot{\sigma}_{i}(t) \leq & \sum_{k} a_{i}^{k} x_{i}(t)-\sum_{k} \int_{t-\tau_{i}^{k}}^{t} a_{i}^{k} \dot{x}_{i}(\eta) \mathrm{d} \eta+\sum_{s} \int_{t-\bar{\tau}_{i}^{s}+\varsigma_{i}}^{t-\bar{\tau}_{i}^{s}} \bar{a}_{i}^{s} \dot{x}_{i}(\eta) \mathrm{d} \eta+\sum_{s} \bar{c}_{i}^{s} x_{i}(t)- \\
& \sum_{s} \int_{t-\bar{\tau}_{i}^{s}+\varsigma_{i}}^{t} \bar{c}_{i}^{s} \dot{x}_{i}(\eta) \mathrm{d} \eta+R_{i}\left(t+\varsigma_{i}\right) .
\end{aligned}
$$

Hence, we obtain the following inequality

$$
\begin{aligned}
\dot{\sigma}_{i}(t) \leq & \left(\sum_{k} a_{i}^{k}+\sum_{s} \bar{c}_{i}^{s}\right) x_{i}(t)+ \\
& \left(\sum_{k}\left|a_{i}^{k}\right| \tau_{i}^{k}+\sum_{s}\left|\bar{a}_{i}^{s}\right| \varsigma_{i}+\sum_{s} \bar{c}_{i}^{s}\left(\bar{\tau}_{i}^{s}-\varsigma_{i}\right)\right)\left\|\dot{x}_{i}(t)\right\|_{L^{\infty}}+\left\|R_{i}(t)\right\|_{L^{\infty}},
\end{aligned}
$$

therefore, $\dot{\sigma}_{i}(t)<0$ if $x_{i}(t)>x_{i}^{d}$ where $x_{i}^{d}$ is given in (17). Thus, we have to verify that indeed $x_{i}(t)>x_{i}^{d}$ when the sliding surface is reached. Note that, when $u_{i}$ switches from $u_{i}(t)=1$ to $u_{i}(t)=0, u_{i}\left(t-\varsigma_{i}\right)=1$. Thus, if $\sigma_{i}(t)=0$, then

$$
0=x_{i}(t)+\varsigma_{i} b_{i}-\sigma_{i}^{*}+\int_{t-\varsigma_{i}}^{t}\left(\sum_{s, r \neq i} u_{r}\left(\eta-\varsigma_{i}^{s, r}+\varsigma_{i}\right) \bar{a}_{i}^{s, r} x_{i}\left(\eta-\bar{\tau}_{i}^{s}+\varsigma_{i}\right)+R_{i}\left(\eta+\varsigma_{i}\right)\right) \mathrm{d} \eta .
$$

From this equation we obtain the inequality

$$
x_{i}(t) \geq-\varsigma_{i} b_{i}+\sigma_{i}^{*}-\int_{t-\varsigma_{i}}^{t} \sum_{s} \bar{c}_{i}^{s} x_{i}\left(\eta-\bar{\tau}_{i}^{s}+\varsigma_{i}\right) \mathrm{d} \eta-\varsigma_{i}\left\|R_{i}(t)\right\|_{L^{\infty}} .
$$

Now, we have proven in Section 5.1 that $x_{i}(t) \leq x_{i}^{*}$ for all $t \leq t_{i}^{*}$ (where $t_{i}^{*}$ is the reaching instant), thus,

$$
x_{i}\left(t_{i}^{*}\right) \geq-\varsigma_{i} b_{i}+\sigma_{i}^{*}-\varsigma_{i} \sum_{s} \bar{c}_{i}^{s} x_{i}^{*}-\varsigma_{i}\left\|R_{i}(t)\right\|_{L^{\infty}} .
$$

From this inequality it is verified that $x_{i}\left(t_{i}^{*}\right)>x_{i}^{d}$ if $x_{i}^{*}>\tilde{x}_{i}$, where $\tilde{x}_{i}$ is given in (17).

2. To finalise the proof of the existence of the sliding mode we need to verify that if $u_{i}$ switches from $u_{i}(t)=0$ to $u_{i}(t)=1$, then $\dot{\sigma}_{i}(t)>0$. In this case, the dynamics of the sliding variable (given by (24)) becomes

$$
\begin{aligned}
\dot{\sigma}_{i}(t)= & \sum_{k} a_{i}^{k} x_{i}\left(t-\tau_{i}^{k}\right)+\sum_{s} \bar{a}_{i}^{s} x_{i}\left(t-\bar{\tau}_{i}^{s}\right)+b_{i}+ \\
& \sum_{s, r \neq i} u_{r}\left(t-\varsigma_{i}^{s, r}+\varsigma_{i}\right) \bar{a}_{i}^{s, r} x_{i}\left(t-\bar{\tau}_{i}^{s}+\varsigma_{i}\right)+R_{i}\left(t+\varsigma_{i}\right),
\end{aligned}
$$


which can be bounded as follows,

$$
\begin{aligned}
\dot{\sigma}_{i}(t) \geq & \sum_{k} a_{i}^{k} x_{i}\left(t-\tau_{i}^{k}\right)+\sum_{s} \bar{a}_{i}^{s} x_{i}\left(t-\bar{\tau}_{i}^{s}\right)+b_{i}-\sum_{s, r \neq i} c_{i}^{s} x_{i}\left(t-\bar{\tau}_{i}^{s}+\varsigma_{i}\right), \\
\dot{\sigma}_{i}(t) \geq & \sum_{k} a_{i}^{k} x_{i}(t)-\sum_{k} a_{i}^{k} \int_{t-\tau_{i}^{k}}^{t} \dot{x}_{i}(\eta) \mathrm{d} \eta+\sum_{s} \bar{a}_{i}^{s} x_{i}(t)- \\
& \sum_{s} \bar{a}_{i}^{s} \int_{t-\bar{\tau}_{i}^{s}}^{t} \dot{x}_{i}(\eta) \mathrm{d} \eta-\sum_{s} c_{i}^{s} x_{i}(t)+\sum_{s} c_{i}^{s} \int_{t-\bar{\tau}_{i}^{s}+\varsigma_{i}}^{t} \dot{x}_{i}(\eta) \mathrm{d} \eta+b_{i} .
\end{aligned}
$$

Thus, we obtain the bound

$$
\begin{aligned}
\dot{\sigma}_{i}(t) \geq & \left(\sum_{k} a_{i}^{k}+\sum_{s}\left(\bar{a}_{i}^{s}-c_{i}^{s}\right)\right) x_{i}(t)+b_{i}- \\
& \left(\sum_{k}\left|a_{i}^{k}\right| \tau_{i}^{k}+\sum_{s}\left|\bar{a}_{i}^{s}\right| \bar{\tau}_{i}^{s}+\sum_{s} c_{i}^{s}\left(\bar{\tau}_{i}^{s}-\varsigma_{i}\right)\right)\left\|\dot{x}_{i}(t)\right\|_{L^{\infty}} .
\end{aligned}
$$

Since $\sum_{k} a_{i}^{k}+\sum_{s}\left(\bar{a}_{i}^{s}-c_{i}^{s}\right)<0$, we can assure that $\dot{\sigma}_{i}(t)>0$ if $x_{i}(t)<\bar{x}_{i}^{*}$ (with $\bar{x}_{i}^{*}$ given by (16)). Such a condition is true if $x_{i}^{*}<\bar{x}_{i}^{*}$, this can be verified by following the same procedure as in Section 5.1

\subsection{Stability of the sliding dynamics}

For the sliding dynamics analysis, first note that, from (21) and (24), the sliding variable can be rewritten as follows

$$
\sigma_{i}(t)=x_{i}(t)-\int_{t-\varsigma_{i}}^{t}\left(\sum_{k} a_{i}^{k} x_{i}\left(\eta-\tau_{i}^{k}\right)+\sum_{s} \bar{a}_{i}^{s} x_{i}\left(\eta-\bar{\tau}_{i}^{s}\right)\right) \mathrm{d} \eta-\sigma_{i}^{*}+\int_{t-\varsigma_{i}}^{t} \dot{\sigma}_{i}(\eta) \mathrm{d} \eta,
$$

or equivalently

$$
\sigma_{i}\left(t-\varsigma_{i}\right)=x_{i}(t)-\int_{t-\varsigma_{i}}^{t}\left(\sum_{k} a_{i}^{k} x_{i}\left(\eta-\tau_{i}^{k}\right)+\sum_{s} \bar{a}_{i}^{s} x_{i}\left(\eta-\bar{\tau}_{i}^{s}\right)\right) \mathrm{d} \eta-\sigma_{i}^{*} .
$$

Note that once the sliding mode has been reached in $t_{i}^{*}$, the state variable $x_{i}(t)$ may be non-constant in sliding mode, however, we know that it remains bounded, see Lemma 4 . Thus, for all $t \geq t_{i}^{*}+\varsigma_{i}$, the dynamics of $x_{i}$ is described by the integral equation

$$
x_{i}(t)-\int_{t-\varsigma_{i}}^{t}\left(\sum_{k} a_{i}^{k} x_{i}\left(\eta-\tau_{i}^{k}\right)+\sum_{s} \bar{a}_{i}^{s} x_{i}\left(\eta-\bar{\tau}_{i}^{s}\right)\right) \mathrm{d} \eta-\sigma_{i}^{*}=0 .
$$

If we define the regulation error $\chi_{i}$ by means of the change of coordinates $\chi_{i}(t)=x_{i}(t)-x_{i}^{*}$, then the error dynamics is given by

$$
\chi_{i}(t)-\int_{t-\varsigma_{i}}^{t}\left(\sum_{k} a_{i}^{k} \chi_{i}\left(\eta-\tau_{i}^{k}\right)+\sum_{s} \bar{a}_{i}^{s} \chi_{i}\left(\eta-\bar{\tau}_{i}^{s}\right)\right) \mathrm{d} \eta=0
$$


or, equivalently,

$$
\chi_{i}(t)-\sum_{k} \int_{t-\varsigma_{i}-\tau_{i}^{k}}^{t-\tau_{i}^{k}} a_{i}^{k} \chi_{i}(\eta) \mathrm{d} \eta-\sum_{s} \int_{t-\varsigma_{i}-\bar{\tau}_{i}^{s}}^{t-\bar{\tau}_{i}^{s}} \bar{a}_{i}^{s} \chi_{i}(\eta) \mathrm{d} \eta=0 .
$$

Note that this equation can be rewritten as follows

$$
\chi_{i}(t)+\int_{t_{i}^{*}+\varsigma_{i}}^{t}\left(\sum_{k} A_{i}^{k}(t-\eta)+\sum_{s} \bar{A}_{i}^{s}(t-\eta)\right) \chi_{i}(\eta) \mathrm{d} \eta=v_{i}\left(t ; t_{i}^{*}+\varsigma_{i}\right),
$$

where the functions $A_{i}^{k}, \bar{A}_{i}^{s}$ are given by 22 (by replacing the parameter $r$ by $t-\eta$ ), and the function $v_{i}:\left[t_{i}^{*}+\varsigma_{i}, \infty\right) \rightarrow \mathbb{R}$ is given by

$$
v_{i}\left(t ; t_{i}^{*}+\varsigma_{i}\right)=-\sum_{k} \int_{\rho_{i}}^{t_{i}^{*}+\varsigma_{i}} A_{i}^{k}(t-\eta) \chi_{i}(\eta) \mathrm{d} \eta-\sum_{s} \int_{\varrho_{i}}^{t_{i}^{*}+\varsigma_{i}} \bar{A}_{i}^{s}(t-\eta) \chi_{i}(\eta) \mathrm{d} \eta,
$$

with $\rho_{i}=\min \left(t_{i}^{*}+\varsigma_{i}, t-\varsigma_{i}-\tau_{i}^{k}\right), k=1, \ldots, N_{i}^{1}$, and $\varrho_{i}=\min \left(t_{i}^{*}+\varsigma_{i}, t-\varsigma_{i}-\bar{\tau}_{i}^{s}\right)$, $s=1, \ldots, N_{i}^{2}$. Note that $v_{i}\left(t ; t_{i}^{*}+\varsigma_{i}\right)=0$ for all $t \geq t_{i}^{*}+2 \varsigma_{i}+\max _{k, s}\left(\tau_{i}^{k}, \bar{\tau}_{i}^{s}\right)$. Moreover, since $x_{i}(t)$ is bounded for all $t$, then $\chi_{i}(t)$ is also bounded. Hence, there exists $\bar{v}_{i} \in \mathbb{R}_{\geq 0}$ such that $\left\|v_{i}\left(t ; t_{i}^{*}+\varsigma_{i}\right)\right\|_{L^{1}} \leq \bar{v}_{i}$.

Since $\sum_{k} A_{i}^{k}(r)+\sum_{s} \bar{A}_{i}^{s}(r)=K_{i}(r)$, we can rewrite (27) as follows

$$
\chi_{i}(t)+\int_{t_{i}^{*}+\varsigma_{i}}^{t} K_{i}(t-\eta) \chi_{i}(\eta) \mathrm{d} \eta=v_{i}\left(t ; t_{i}^{*}+\varsigma_{i}\right),
$$

which is a Volterra integral equation of the second kind with a convolution kernel $K_{i}$.

Since $K_{i}$ is a measurable kernel, according to Lemma 8 (see Appendix A.2), condition (23) ensures that $K_{i}$ is a kernel of type $L^{1}$, furthermore, it has a resolvent $\mathcal{R}_{i}$ of type $L^{1}$. Thus, since $v_{i} \in L^{1}$, Lemma 7 (see Appendix A.2) ensures the existence of a unique solution $\chi_{i}$ of $(29)$ such that $\chi_{i} \in L^{1}$ and it is given by

$$
\chi_{i}(t)=v_{i}\left(t ; t_{i}^{*}+\varsigma_{i}\right)-\int_{t_{i}^{*}+\varsigma_{i}}^{t} \mathcal{R}_{i}(t-\eta) v_{i}\left(\eta ; t_{i}^{*}+\varsigma_{i}\right) \mathrm{d} \eta .
$$

Finally, since $K_{i}$ is a convolution kernel, Lemma 9 (see Appendix A.2) guarantees that $\chi_{i}(t)$ tends to zero exponentially as $t$ tends to infinity. Thus, $x_{i}(t)$ tends exponentially to the set point $x_{i}^{*}$.

\section{Robustness}

In this section we consider the presence of additive disturbances in (2), i.e.

$$
\dot{x}_{i}(t)=F_{i}(x, u)+\delta_{i}(t),
$$

where the functional $F_{i}$ denotes the right-hand side of $(2)$, and each $\delta_{i} \in L^{\infty}\left(\mathbb{R}_{\geq t_{0}}\right)$ 
Remark 5. The functions $\delta_{i}$ represent external disturbances, nonetheless, they can also represent uncertainty in the model. This is possible due to the boundedness property of the solution of the system.

According to the nature of the physical system, we can make the following assumptions.

Assumption 3. The disturbance $\delta=\left[\delta_{1}, \ldots, \delta_{n}\right]^{\top}$ is such that the solutions of (30) are nonnegative, and $\left\|\delta_{i}(t)\right\|_{L^{\infty}(\mathbb{R})}=\Delta_{i}<b_{i}, i=1, \ldots, n$, for some $\Delta_{i} \in \mathbb{R}_{>0}$.

The following theorem proves ISS (input-to-state stability) property [20] of the disturbed closed-loop system (30).

Theorem 2. Consider (30) with (3). Suppose that the assumptions of Theorem 1 hold. For any disturbance $\delta$ satisfying Assumption 3 , the regulation error $x(t)-x^{*}$ is ultimately bounded, and its ultimate bound $\beta \in \mathbb{R}_{\geq 0}$ is such that $\beta \rightarrow 0$ as $\max _{i} \Delta_{i} \rightarrow 0$.

Proof. Note that Lemma 2 and the essential boundedness of the disturbance guarantee that the solution of (30) is also bounded. Indeed, the following bounds are obtained by means of the same procedure adopted in the proof of Lemma 4 .

$$
\left\|x_{i}(t)\right\|_{L^{\infty}} \leq z_{i} \max _{j} \tilde{B}_{j}
$$

where $z_{i}$ is as defined in (5), $\tilde{B}_{i}=2 b_{i}+\sum_{j \neq i} b_{i j}$ for $R_{i}$ as in Case 2, and $\tilde{B}_{i}=2 b_{i}+$ $\left\|R_{i}(t)\right\|_{L^{\infty}}$ for $R_{i}$ as in Case 1. Observe that, since $x(t)$ is bounded, each $\sigma_{i}$ is also bounded for all $t \in \mathbb{R}$, this is clear from the definition of $\sigma_{i}$ in (21).

Although, this is enough to prove ultimate boundedness we want to prove, furthermore, that the steady state regulation error is small for small disturbances. Thus, note that by means of an analogous analysis to that made in Section 5.1, it can be verified that for each $x_{i}^{*} \in\left(\underline{x}_{i}^{*}, \bar{x}_{i}^{*}\right)$ there exists a sufficiently small $\Delta_{i}$ such that the sliding motion is established despite the presence of disturbances. Nonetheless, the disturbance still affects the dynamics on the sliding motion. Let us analyse in detail this case. The time derivative of the sliding variable is given by

$$
\begin{gathered}
\dot{\sigma}_{i}(t)=\sum_{k} a_{i}^{k} x_{i}\left(t-\tau_{i}^{k}\right)+\sum_{s} \bar{a}_{i}^{s} x_{i}\left(t-\bar{\tau}_{i}^{s}\right)-\left(1-u_{i}(t)\right) \sum_{s} \bar{a}_{i}^{s} x_{i}\left(t-\bar{\tau}_{i}^{s}+\varsigma_{i}\right)+ \\
b_{i} u_{i}(t)+\sum_{s, r \neq i} u_{r}\left(t-\varsigma_{i}^{s, r}+\varsigma_{i}\right) \bar{a}_{i}^{s, r} x_{i}\left(t-\bar{\tau}_{i}^{s}+\varsigma_{i}\right)+R_{i}\left(t+\varsigma_{i}\right)+\delta_{i}(t) .
\end{gathered}
$$

In the nominal case $(\delta=0)$ the sliding variable can be written as (26), thus, for the disturbed case (31) we have that

$\sigma_{i}(t)=x_{i}(t)-\int_{t-\varsigma_{i}}^{t}\left(\sum_{k} a_{i}^{k} x_{i}\left(\eta-\tau_{i}^{k}\right)+\sum_{s} \bar{a}_{i}^{s} x_{i}\left(\eta-\bar{\tau}_{i}^{s}\right)\right) \mathrm{d} \eta-\sigma_{i}^{*}+\int_{t-\varsigma_{i}}^{t}\left(\dot{\sigma}_{i}(\eta)-\delta_{i}(\eta)\right) \mathrm{d} \eta$, 
or equivalently,

$$
\sigma_{i}\left(t-\varsigma_{i}\right)+\bar{\delta}_{i}(t)=x_{i}(t)-\int_{t-\varsigma_{i}}^{t}\left(\sum_{k} a_{i}^{k} x_{i}\left(\eta-\tau_{i}^{k}\right)+\sum_{s} \bar{a}_{i}^{s} x_{i}\left(\eta-\bar{\tau}_{i}^{s}\right)\right) \mathrm{d} \eta-\sigma_{i}^{*}
$$

where $\bar{\delta}_{i}(t):=\int_{t-\varsigma_{i}}^{t} \delta_{i}(\eta) \mathrm{d} \eta$. Considering again the change of coordinates $\chi_{i}(t)=x_{i}(t)-x_{i}^{*}$, and by using the same procedure as in Section 5.3, we obtain from (32) the integral equation

$$
\chi_{i}(t)+\int_{t_{0}}^{t} K_{i}(t-\eta) \chi_{i}(\eta) \mathrm{d} \eta=w_{i}(t)
$$

where $w_{i}(t):=\sigma_{i}\left(t-\varsigma_{i}\right)+\bar{\delta}_{i}(t)+v_{i}\left(t ; t_{0}\right)$, and $v_{i}$ is given by $(28)$. Observe that $\left\|\bar{\delta}_{i}(t)\right\|_{L^{\infty}} \leq$ $\Delta_{i} \varsigma_{i}$, and (by using the same arguments as in Section 5.3) we assert that $v_{i} \in L^{1} \cap L^{\infty}$, hence, $w_{i} \in L^{\infty}$. Now, we have proven that the kernel $K_{i}$ has a resolvent $\mathcal{R}_{i}$ of type $L^{1}$, thus, according to Lemma 7 (see Appendix A.2) the solution $\chi_{i}$ of (33) is such that $\chi_{i} \in L^{\infty}$, and

$$
\chi_{i}(t)=w_{i}(t)-\int_{t_{0}}^{t} \mathcal{R}_{i}(t-\eta) w_{i}(\eta) \mathrm{d} \eta .
$$

Assume that $\Delta_{i}$ is sufficiently small such that the sliding motion is established at some $t=t_{i}^{*}$, thus, for $t \geq t_{i}^{*}+\varsigma_{i}$ we have that $w_{i}(t)=\bar{\delta}_{i}(t)+v_{i}\left(t ; t_{i}^{*}+\varsigma_{i}\right)$, and

$$
\chi_{i}(t)=\bar{\delta}_{i}(t)+v_{i}\left(t ; t_{i}^{*}+\varsigma_{i}\right)-\int_{t_{i}^{*}+\varsigma_{i}}^{t} \mathcal{R}_{i}(t-\eta)\left(\bar{\delta}_{i}(\eta)+v_{i}\left(\eta ; t_{i}^{*}+\varsigma_{i}\right)\right) \mathrm{d} \eta
$$

Since $v_{i}\left(t ; t_{i}^{*}+\varsigma_{i}\right)=0$ for all $t \geq t_{i}^{*}+2 \varsigma_{i}+\max _{k, s}\left(\tau_{i}^{k}, \bar{\tau}_{i}^{s}\right)$, and $\lim _{t \rightarrow \infty} \int_{t_{i}^{*}+\varsigma_{i}}^{t} \mathcal{R}_{i}(t-\eta) v_{i}\left(\eta ; t_{i}^{*}+\right.$ $\left.\varsigma_{i}\right) \mathrm{d} \eta=0$, we have that

$$
\chi_{i}(t) \rightarrow \bar{\delta}_{i}(t)-\int_{t_{i}^{*}+\varsigma_{i}}^{t} \mathcal{R}_{i}(t-\eta) \bar{\delta}_{i}(\eta) \mathrm{d} \eta
$$

as $t \rightarrow \infty$. But note that $\int_{t_{i}^{*}+\varsigma_{i}}^{t} \mathcal{R}_{i}(t-\eta) \bar{\delta}_{i}(\eta) \mathrm{d} \eta=\int_{t_{i}^{*}+\varsigma_{i}}^{t} \mathcal{R}_{i}(\eta) \bar{\delta}_{i}(t-\eta) \mathrm{d} \eta$, hence,

$$
\bar{\delta}_{i}(t)-\int_{t_{i}^{*}+\varsigma_{i}}^{t} \mathcal{R}_{i}(t-\eta) \bar{\delta}_{i}(\eta) \mathrm{d} \eta \leq\left(1+\left\|\mathcal{R}_{i}(t)\right\|_{L^{1}}\right) \Delta_{i} \varsigma_{i} .
$$

Therefore, the ultimate bound for $\chi_{i}$ is proportional to $\Delta_{i}$.

\section{Numerical Example}

In this section we provide an academic example to simulate the developed control scheme. Consider (2) with $n=2, N_{i}^{1}=2, N_{i}^{2}=2$, and $N_{i}^{3}=1$. The parameters of the system are 


\begin{tabular}{c|c|c|c|c|c|c|c|c|c|c}
\hline$i$ & $a_{i}^{1}$ & $\tau_{i}^{1}$ & $a_{i}^{2}$ & $\tau_{i}^{2}$ & $\bar{a}_{i}^{1}$ & $\bar{\tau}_{i}^{1}$ & $\bar{a}_{i}^{2}$ & $\bar{\tau}_{i}^{2}$ & $b_{i}$ & $\varsigma_{i}$ \\
\hline \hline 1 & -4.6 & 0.005 & 1.8 & 0.02 & -1.3 & 0.02 & 1.1 & 0.03 & 2.2 & 0.015 \\
\hline 2 & -4.3 & 0.005 & 1.6 & 0.022 & -1.2 & 0.022 & 1 & 0.03 & 2 & 0.017 \\
\hline
\end{tabular}

Table 1: Parameters for the system.

\begin{tabular}{c|c|c|c|c|c|c|c|c|c|c|c}
\hline $\bar{a}_{1}^{1,2}$ & $\bar{a}_{1}^{2,2}$ & $\varsigma_{1}^{1,2}$ & $\bar{a}_{12}^{s^{\prime}, 1}$ & $\bar{\tau}_{12}^{s^{\prime}}$ & $\varsigma_{12}^{s^{\prime}, 1}$ & $a_{12}^{k^{\prime}}$ & $\tau_{12}^{k^{\prime}}$ & $\bar{a}_{12}^{s^{\prime}, 2}$ & $\varsigma_{12}^{s^{\prime}, 2}$ & $b_{12}$ & $\varsigma_{12}$ \\
\hline \hline 0.15 & -0.15 & 0.06 & 0.12 & 0.05 & 0.09 & 0.15 & 0.03 & 0.12 & 0.09 & 0.1 & 0.09 \\
\hline
\end{tabular}

Table 2: Parameters for the coupling terms of the subsystem $i=1$.

given in Tables 1.3. These parameters have been chosen in a similar order of magnitude as those given in [6] for the SISO case. We have also chosen $\varsigma_{i}^{1, r}=\varsigma_{i}^{2, r}$.

Such a set of parameters satisfy Lemmas 1 and 2 . For Lemma 1, the parameters satisfy the conditions 1(b). Furthermore, we obtain the intervals

$$
\left(\underline{x}_{1}^{*}, \bar{x}_{1}^{*}\right)=(0.324,0.574), \quad\left(\underline{x}_{2}^{*}, \bar{x}_{2}^{*}\right)=(0.308,0.542) .
$$

Thus, for the simulation we choose the reference points $x_{1}^{*}=0.5$, and $x_{2}^{*}=0.4$. Since (23) is satisfied, the convergence is guaranteed by Theorem 1 . The simulation is carried out with MATLAB by using an explicit Euler integration with an integration step of $0.1 \mathrm{~ms}$. First, we describe the simulation for the nominal case (without disturbances). In Fig. 4 we can see both sliding variables converging to zero in finite-time. The control signals, displaying sliding regimes, are shown in Fig. 5. Fig. 6 shows the transient of the system's states from the zero-initial condition to the steady-state. The evolution of the states in sliding regime can be appreciated in Fig. 7. Note that the behaviour of the sliding variables is similar to that of the states of the system (see Fig. 4 and Fig. 6), this reflects the similarity between the equations describing the system and the sliding dynamics.

Now, for the simulation of the disturbed case, we choose the functions $\delta_{1}(t)=\frac{2}{3}(1+$ $\sin (35 t)+\sin (45 t))$, and $\delta_{2}(t)=\frac{1}{6}(1+\sin (35 t)-\sin (50 t) / 2)$. Fig. 8 shows the states of the system evolving from the zero-initial conditions to a neighbourhood of the reference points. The effect of the disturbances on the states can be appreciated in Fig. 9. Observe that the states remain bounded and close to the reference points, confirming this way, the robustness of the control scheme. Note that, the magnitude of $\delta_{2}$ is less than the magnitude of $\delta_{1}$, thus, the state $x_{2}$ is affected in a lesser extent by the disturbances than the state $x_{1}$, see Fig. 9. In fact, the sliding regime is maintained for $x_{2}$ despite the disturbance, while

\begin{tabular}{c|c|c|c|c|c|c|c|c|c|c|c}
\hline $\bar{a}_{2}^{1,1}$ & $\bar{a}_{2}^{2,1}$ & $\varsigma_{2}^{1,1}$ & $\bar{a}_{21}^{s^{\prime}, 2}$ & $\bar{\tau}_{21}^{s^{\prime}}$ & $\varsigma_{21}^{s^{\prime}, 2}$ & $a_{21}^{k^{\prime}}$ & $\tau_{21}^{k^{\prime}}$ & $\bar{a}_{21}^{s^{\prime}, 1}$ & $\varsigma_{21}^{s^{\prime}, 1}$ & $b_{21}$ & $\varsigma_{21}$ \\
\hline \hline 0.12 & -0.12 & 0.06 & 0.1 & 0.06 & 0.08 & 0.13 & 0.03 & 0.1 & 0.08 & 0.11 & 0.08 \\
\hline
\end{tabular}

Table 3: Parameters for the coupling terms of the subsystem $i=2$. 

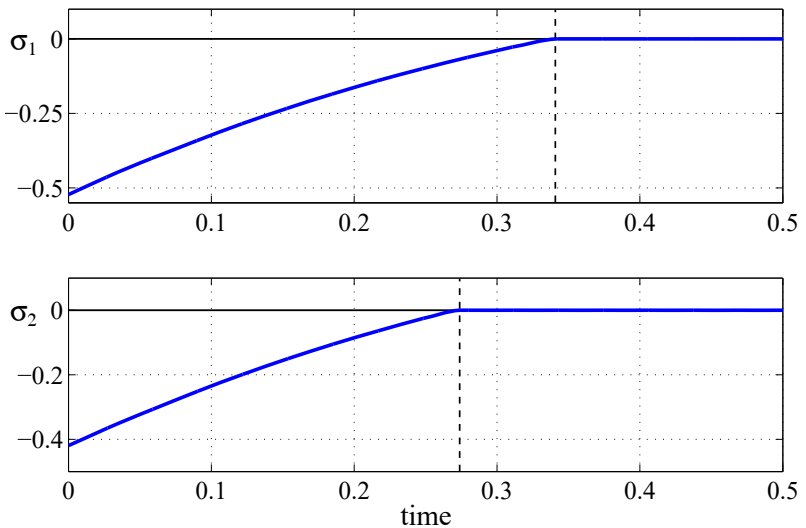

Figure 4: Sliding variables (nominal case), the vertical dashed line indicates the reaching time.

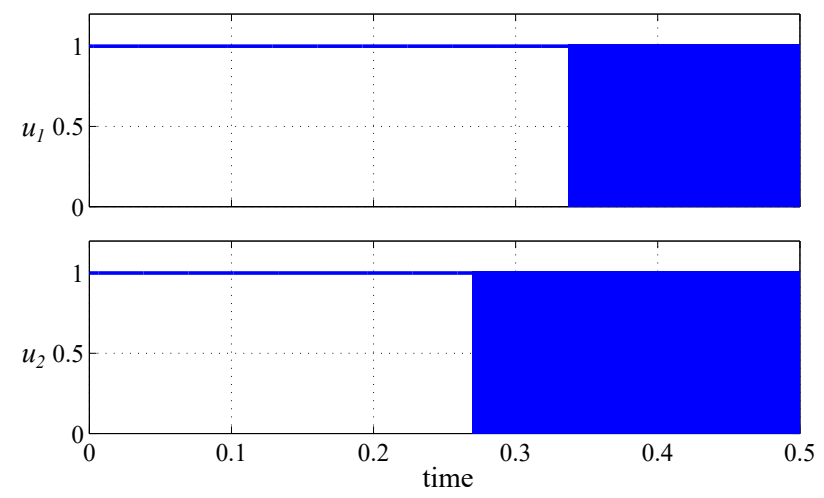

Figure 5: Control signals (nominal case) exhibiting the establishment of the sliding regimes in both subsystems.

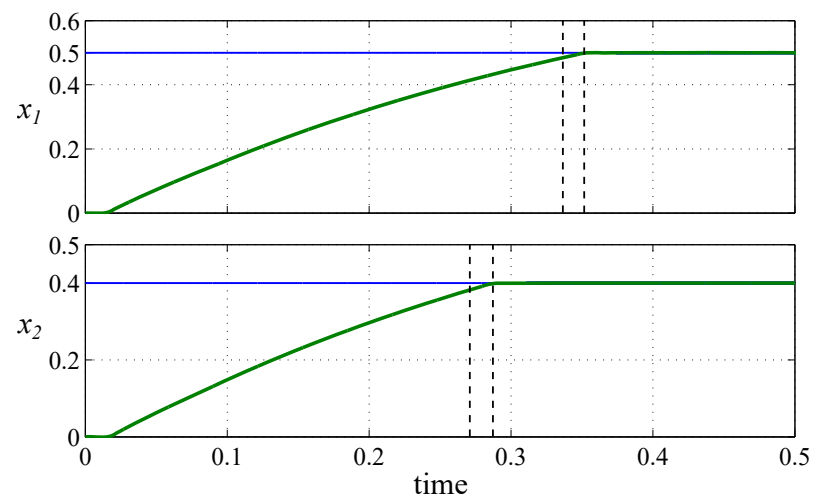

Figure 6: States of the system (nominal case). The time elapsed between the dashed lines corresponds to the input delay. 

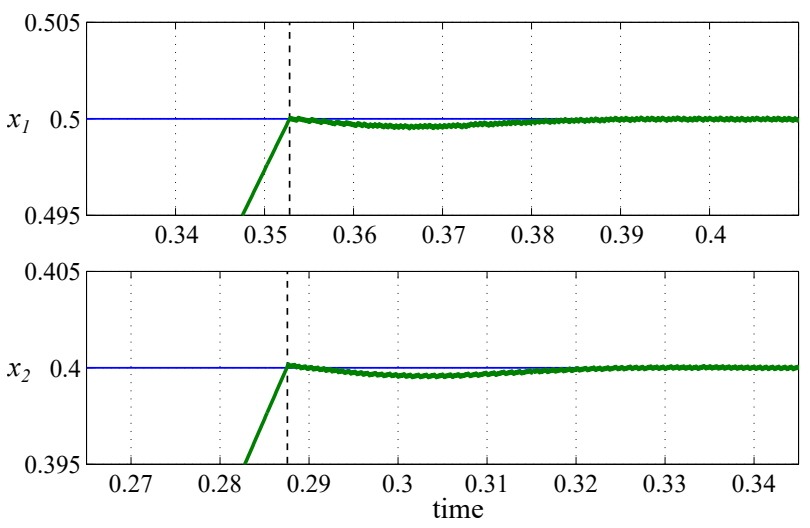

Figure 7: Close-up of the states evolving in the sliding regimes to the steady-state behaviour (nominal case).
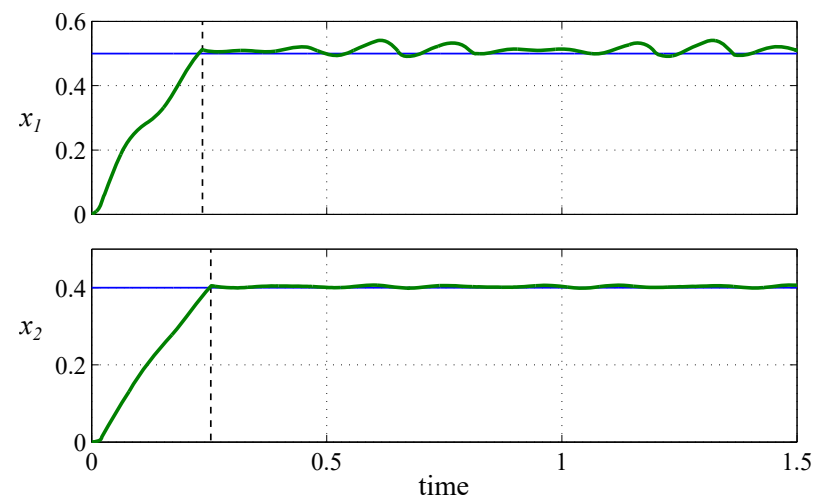

Figure 8: States of the system (disturbed case).

for $x_{1}$ the sliding regime is established intermittently, this is shown in Fig. 10 and Fig. 11 . The control signals are shown in Fig. 12 , there, we can see how the sliding motion behaves intermittent for the first state due to the effect of the disturbance.

\section{Conclusion}

Motivated as in [7] by active flow control issues, the extension of a control strategy for nonlinear (bilinear) systems with multiple delays from MIMO to SISO models has been presented. Accordingly to the on-off feature of usual air-jet actuators, we have focused on the development of sliding mode control. The main advantages of the controller are its simplicity and its robustness properties. Although the sliding variable design is inspired by the prediction technique, no state predictor is used in the control scheme. This allows to avoid a high computational cost in the control implementation.

The major drawbacks of the design technique are: 1) Since the bounds of the system's 

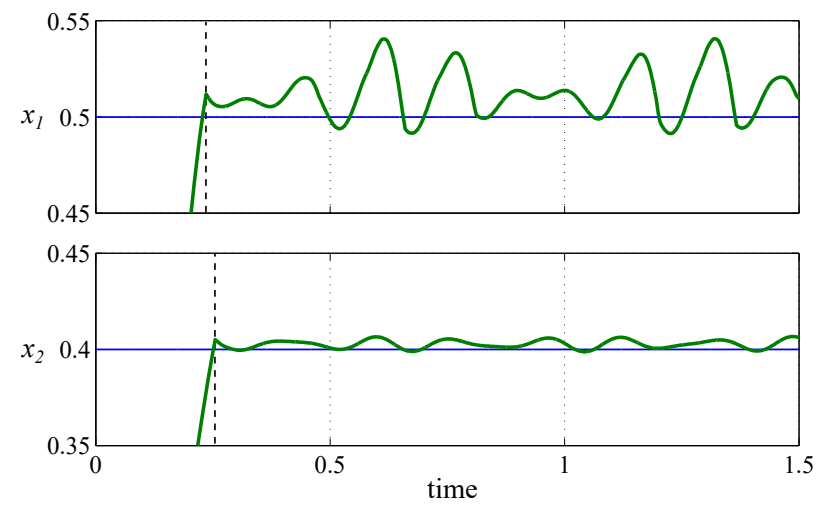

Figure 9: Close-up of the states (disturbed case) confined to a neighbourhood of the reference points.
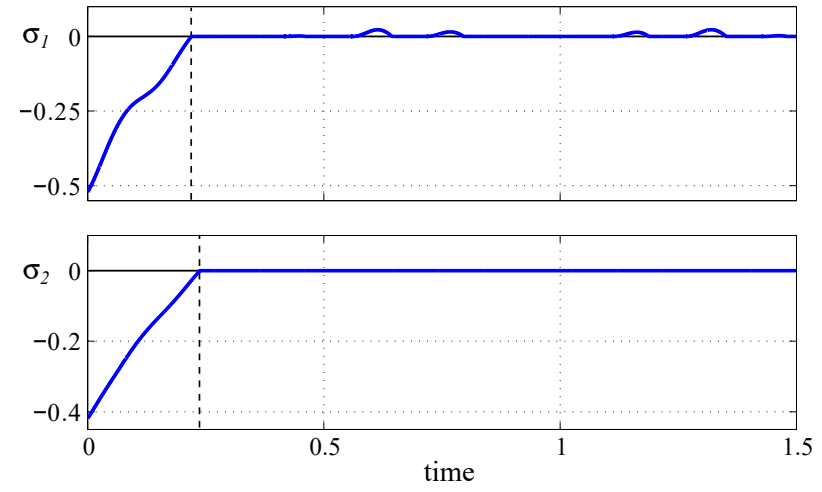

Figure 10: Sliding variables (disturbed case). The sliding motion is established for $\sigma_{2}$ despite the disturbance.
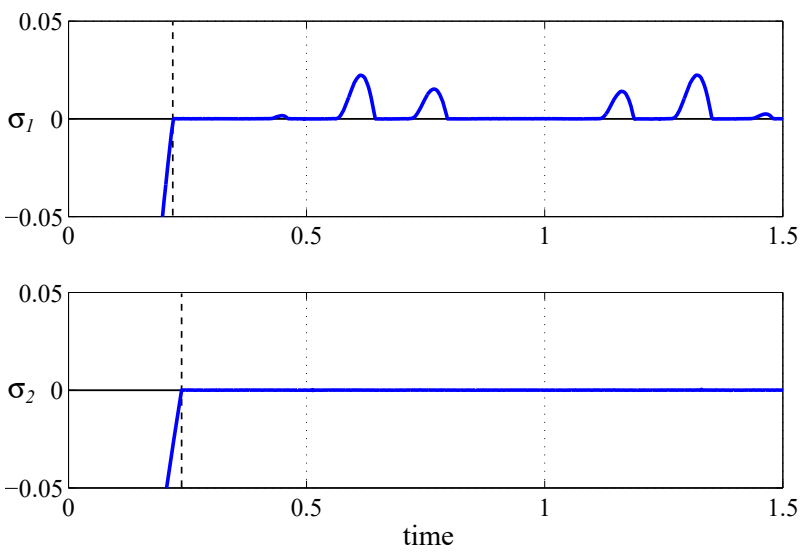

Figure 11: Close-up of the sliding variables (disturbed case). 

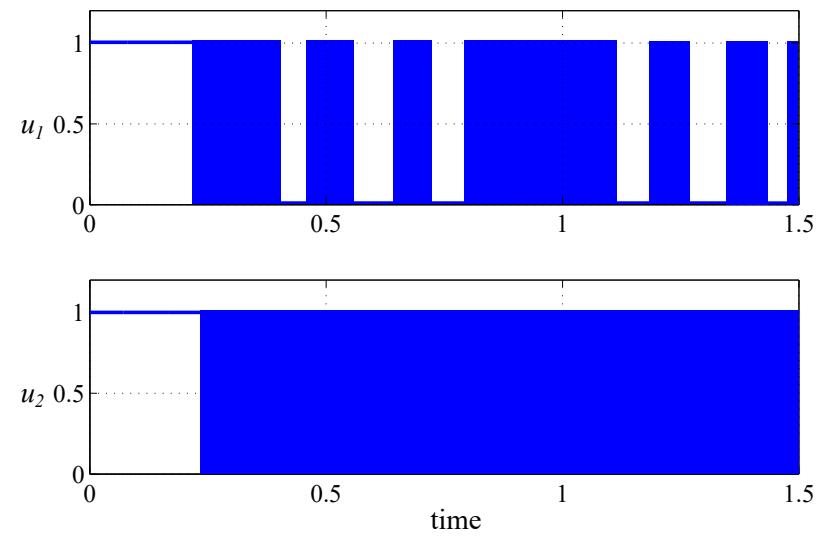

Figure 12: Control signals (disturbed case). An intermittent sliding regime can be observed for the first subsystem.

state, and the range for the operation points, were computed for the general case, their resulting values can be quite restrictive; Nevertheless, the proof of Theorem 1 indicates the general procedure for finding the conditions that guarantee the efficacy of the controller. Thus, all the bounds and conditions could be improved by means of a thorough analysis in each particular case; 2) There is no clear methodology to propose different sliding variables and to prove the establishment of the sliding regime; 3) It is not straightforward to determine how restrictive is the condition (23) to guarantee asymptotic stability of the sliding motion.

Ongoing and future work should concern: 1) A current research, fundamental for this investigation, is the experimental validation of the proposed MIMO model. 2) The sliding variable design and the reaching analysis made in this paper slightly differ from those in the works of Feingesicht et al. [6] and Sanchez et al. [19] Thus, it would be interesting to develop a more general procedure to design such a kind of sliding variables and a more efficient method to prove the stability and performance features of this kind of controllers. 3) It would be interesting to establish an adequate framework of comparison between the control scheme developed in this paper and that one in [18].

\section{Acknowledgements}

This work was carried out within the framework of the CNRS Research Federation on Ground Transports and Mobility, in articulation with the ELSAT2020 project supported by the European Community, the French Ministry of Higher Education and Research, the Hauts de France Regional Council. 


\section{References}

[1] R. P. Agarwal, L. Berezansky, E. Braverman, and A. Domoshnitsky. Nonoscillation Theory of Functional Differential Equations with Applications. Springer-Verlag, New York, 2012.

[2] S. L. Brunton and B. R. Noack. Closed-Loop Turbulence Control: Progress and Challenges. ASME Applied Mechanics Reviews, 67(5):1-48, 2015.

[3] C. Chovet, M. Feingesicht, B. Plumejeau, S. Delprat, M. Lippert, L. Keirsbulck, A. Polyakov, J.-P. Richard, and F. Kerhervé. Reducing Car Consumption by Means of a Closed-loop Drag Control. In VEHICULAR 2018, The 7th IARIA Int. Conf. on Advances in Vehicular Systems, Technologies and Applications, June 2018.

[4] A. Domoshnitsky and E. Fridman. A positivity-based approach to delay-dependent stability of systems with large time-varying delays. Systems \& Control Letters, 97:139$148,2016$.

[5] M. Feingesicht. Nonlinear active control of turbulent separated flows: Theory and experiments. PhD thesis, Centrale Lille, France, 2017.

[6] M. Feingesicht, A. Polyakov, F. Kerhervé, and J.-P. Richard. SISO model-based control of separated flows: Sliding mode and optimal control approaches. International Journal of Robust and Nonlinear Control, 27(18):5008-5027, 2017.

[7] M. Feingesicht, C. Raibaudo, A. Polyakov, F. Kerhervé, and J.-P. Richard. A bilinear input-output model with state-dependent delay for separated flow control. In 2016 European Control Conference (ECC), pages 1679-1684, June 2016.

[8] A. F. Filippov. Differential Equations with Discontinuous Righthand Sides. Kluwer. Dordrecht, The Netherlands, 1988.

[9] G. Gripenberg, S.-O. Londen, and O. Staffans. Volterra Integral and Functional Equations. Cambridge University Press, Cambridge, 1990.

[10] J. K. Hale and S. M. Verduyn Lunel. Introduction to Functional Differential Equations. Springer-Verlag, New York, 1993.

[11] Z. Kader, C. Fiter, L. Hetel, and L. Belkoura. Stabilization of switched affine systems with disturbed state-dependent switching laws. International Journal of Robust and Nonlinear Control, 28(2):582-595, 2018.

[12] V. Kolmanovskii and A. Myshkis. Introduction to the Theory and Applications of Functional Differential Equations. Springer, Dordrecht, Netherlands, 1999.

[13] R. McCallen, F. Browand, and J. Ross, editors. The Aerodynamics of Heavy Vehicles: Trucks, Buses, and Trains. Springer-Verlag, Berlin, 2004. 
[14] R. R. Mohler. Bilinear Control Processes: With Applications to Engineering, Ecology, and Medicine. Academic Press, New York, 1973.

[15] P. M. Pardalos and V. A. Yatsenko. Optimization and Control of Bilinear Systems. Springer, New York, 2008.

[16] A. Polyakov and L. Fridman. Stability notions and Lyapunov functions for sliding mode control systems. Journal of the Franklin Institute, 351(4):1831-1865, 2014.

[17] H. L. Royden. Real Analysis. Macmillan Publishing Company, New York, USA, 3rd. edition, 1988.

[18] T. Sanchez, A. Polyakov, E. Fridman, and L. Hetel. A Switching Controller for a class of MIMO Bilinear Systems with Time-Delay. IEEE Transactions on Automatic Control, pages 1-7, 2019.

[19] T. Sanchez, A. Polyakov, J.-P. Richard, and D. Efimov. A robust Sliding Mode Controller for a class of SISO bilinear delayed systems. In 15th International Workshop on Variable Structure Systems (VSS), pages 126-131, July 2018.

[20] E. D. Sontag and Y. Wang. On characterizations of the input-to-state stability property. Systems \& Control Letters, 24(5):351-359, 1995.

[21] Y. Wang, H. R. Karimi, H. Shen, Z. Fang, and M. Liu. Fuzzy-Model-Based Sliding Mode Control of Nonlinear Descriptor Systems. IEEE Transactions on Cybernetics, 49(9):3409-3419, Sep. 2019.

[22] X. Yang and L.-K. Chen. Stability of Discrete Bilinear Systems with Time-Delayed Feedback Functions. IEEE Transactions on Automatic Control, 38(1):158-163, Jan 1993.

\section{A Auxiliary concepts and results}

\section{A.1 Cauchy Matrix}

For a finite $N \in \mathbb{Z}_{>0}$, consider the system

$$
\dot{x}(t)=\sum_{i=1}^{N} A_{i}(t) x\left(t-\tau_{i}\right), \quad x(t) \in \mathbb{R}^{n} .
$$

Assumption 4. Each $\tau_{i} \in \mathbb{R}_{\geq 0}$, and each element of the matrices $A_{i}$ is a Lebesguemeasurable and locally essentially bounded function.

Definition 2 (Cauchy matrix, see e.g., page 466 of Agarwal et al. [1]). The $n \times n$ matrix function $C(t, s)$ that satisfies, for each $s \geq 0$, the problem

$$
\frac{\mathrm{d}}{\mathrm{d} t} C(t, s)=\sum_{i=1}^{N} A_{i}(t) C\left(t-\tau_{i}, s\right), \quad \forall t \in \mathbb{R}_{\geq s},
$$

$C(t, s)=0$ for all $t<s$, and $C(s, s)=I$ ( $I$ is the identity matrix), is called the Cauchy matrix (or fundamental function) of 35 . 
Now consider the system

$$
\dot{x}(t)=\sum_{i=1}^{N} A_{i}(t) x\left(t-\tau_{i}\right)+f(t), \quad x(t) \in \mathbb{R}^{n},
$$

with initial conditions $x(t)=0$ for all $t<0$ and $x(0)=x_{0}$, for some $x_{0} \in \mathbb{R}$.

Lemma 5 (See e.g., page 466 of Agarwal et al. [1]). Consider (36) with $A_{i}$ and $\tau_{i}$ satisfying Assumption 4. If $f: \mathbb{R} \rightarrow \mathbb{R}^{n}$ is a Lebesgue-measurable locally essentially bounded function, then there exists a unique solution of (36) and it can be written as

$$
x(t)=C(t, 0) x_{0}+\int_{0}^{t} C(t, s) f(s) \mathrm{d} s .
$$

Lemma 6. If the differential equation

$$
\dot{z}(t)=\sum_{k=1}^{N} a_{k} z\left(t-\tau_{k}\right), \quad z(t) \in \mathbb{R},
$$

is positive and exponentially stable, then the solution $z(t)$ of the initial value problem

$$
\dot{z}(t)=\sum_{k=1}^{N} a_{k} z\left(t-\tau_{k}\right)+b, \quad z(t)=0, t \leq 0
$$

with $b \in \mathbb{R}_{\geq 0}$, is such that $\lim _{t \rightarrow \infty} z(t)=\bar{z}$, with $\bar{z}=b\left(-\sum_{k=1}^{N} a_{k}\right)^{-1}$.

Proof. Since (37) is exponentially stable, there exist $\alpha, \beta \in \mathbb{R}_{>0}$ such that (according to Lemma 5) the solution of (38) satisfies

$$
|z(t)| \leq \int_{0}^{t} \beta \mathrm{e}^{-\alpha(t-\nu)} b \mathrm{~d} \nu=\frac{\beta}{\alpha} b\left[1-\mathrm{e}^{-\alpha t}\right] .
$$

Now, since (37) is positive and $b$ is a nonnegative constant, Lemma 5 ensures that the solution of (38) is positive and bounded. Moreover, positivity and exponential stability guarantee that, if $t \rightarrow \infty$, then $\dot{z}(t) \rightarrow 0$. Hence $z(t) \rightarrow \bar{z}$ for some constant $\bar{z} \in \mathbb{R}_{\geq 0}$, therefore, $0=\lim _{t \rightarrow \infty} z(t)=\lim _{t \rightarrow \infty}\left(\sum_{k=1}^{N} a_{k} z\left(t-\tau_{k}\right)+b\right)=\sum_{k=1}^{N} a_{k} \bar{z}+b$.

\section{A.2 Volterra equations}

Most of the results recalled in this section can be found in the book of Gripenberg et al., 9] some of them have been simplified for our particular needs.

Consider the integral equation $z(t)+\int_{t_{0}}^{t} k(t, s) z(s) \mathrm{d} s=f(t), t \in J=\mathbb{R}_{\geq t_{0}}$, where $z, f: J \rightarrow \mathbb{R}^{n}$. The kernel $k$ is assumed to be of convolution type, i.e. $k(t, s)=k(t-s)$, thus $k$ can be defined by means of the function $k: J \rightarrow \mathbb{R}$. This integral equation can be rewritten as follows

$$
z(t)+(k * z)(t)=f(t),
$$

where $k * z$ denotes the convolution map $t \mapsto \int_{t_{0}}^{t} k(t-s) z(s) \mathrm{d} s$. A function $r: J \rightarrow \mathbb{R}$ is called a resolvent of (39) if $z(t)=f(t)-(r * f)(t)$. 
Lemma 7 (Theorem 2-2.2 and Theorem 9-3.6 in Gripenberg et al. 9]). Suppose that $k \in L^{1}(J)$ is a convolution kernel that has a resolvent $r \in L^{1}(J)$. If $f \in L^{1}(J)\left(f \in L^{\infty}(J)\right.$, respectively), then (39) has a unique solution $z \in L^{1}(J)\left(z \in L^{\infty}(J)\right.$, respectively) given by $z(t)=f(t)-(r * f)(t)$. Moreover, $\|r * f\|_{L^{1}(J)} \leq\|r\|_{L^{1}(J)}\|f\|_{L^{1}(J)}\left(\|r * f\|_{L^{\infty}(J)} \leq\right.$ $\|r\|_{L^{1}(J)}\|f\|_{L^{\infty}(J)}$, respectively).

Lemma 8 (Corollary 9-3.10, 9]). If $k \in L^{1}(J)$ is a convolution kernel such that $\|k(t)\|_{L^{1}(J)}<$ 1 , then it has a resolvent $r \in L^{1}(J)$.

For the following lemma let us denote the Laplace transform of $k(t)$ as $\hat{k}(z), z \in \mathbb{C}$.

Lemma 9 (Theorem 2-4.1 in Gripenberg et al. [9]). Let $k \in L^{1}\left(\mathbb{R}_{\geq 0}\right)$ be a convolution kernel. The resolvent $r$ is in $L^{1}\left(\mathbb{R}_{\geq 0}\right)$ if and only if $\operatorname{det}(I+\hat{k}(z)) \neq 0$ for all $z \in \mathbb{C}$ such that $\operatorname{Re}\{z\} \geq 0$. 